Aus der Abteilung für Orthopädie und Traumatologie

(Chefarzt: Prof. Dr. med. R. Haaker)

des St. Vincenz Hospitals Brakel

Akademisches Lehrkrankenhaus der Universität Göttingen

\title{
FUNKTIONELLE ERGEBNISSE \\ NACH OBERFLÄCHENERSATZ DES HUMERUSKOPFES \\ DURCH EINE DUROM CUP PROTHESE \\ BEI PATIENTEN MIT EINER OMARTHROSE
}

\author{
INAUGURAL-DISSERTATION \\ zur Erlangung des Doktorgrades \\ der Medizinischen Fakultät der \\ Georg-August-Universität zu Göttingen
}

\author{
vorgelegt von \\ Fouzi Saad \\ aus \\ Damaskus
}

Göttingen 2012 
Dekan:

Prof. Dr. med. M. Schön

I. Berichterstatter: Prof. Dr. med. R. Haaker

II. Berichterstatter: Priv.-Doz. Dr. med. Dumont

III. Berichterstatter:

Tag der mündlichen Prüfung: 13.02.2013 


\section{Inhaltsverzeichnis}

1 EINLEITUNG UND FRAGESTELLUNG .................................................... 1

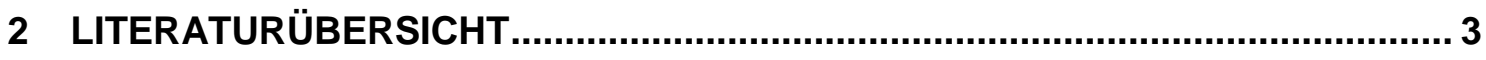

2.1 Vorbemerkung zur Geschichte des Schultergelenksersatzes....................... 3

2.2 Formen und Einteilung der Schultergelenksendoprothesen.......................... 4

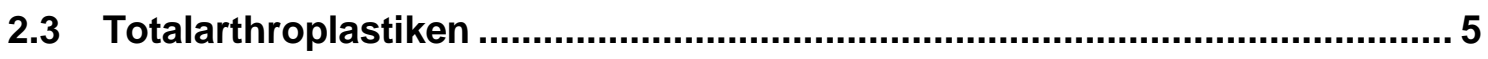

2.4 Hemiarthroplastiken ............................................................................ 7

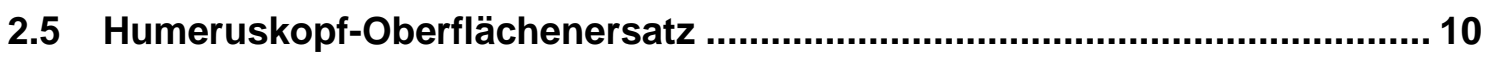

2.5.1 Entwicklung und gebräuchliche Modelle ............................................ 10

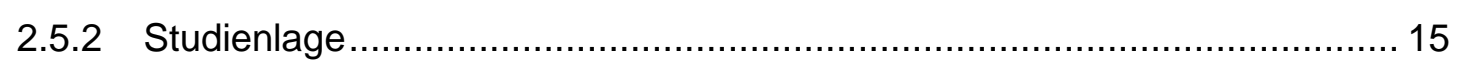

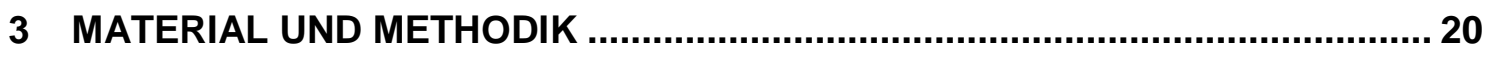

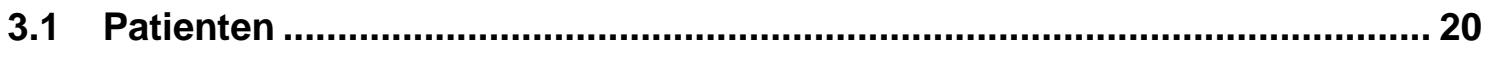

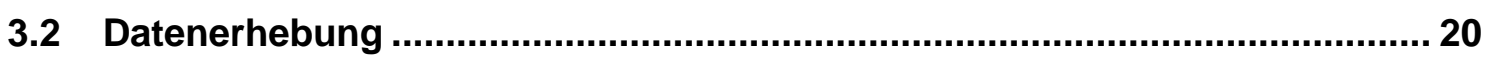

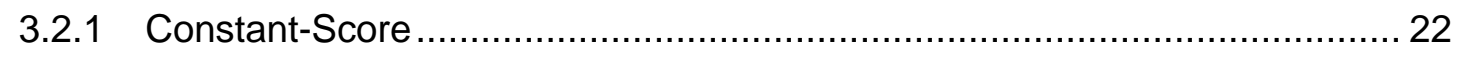

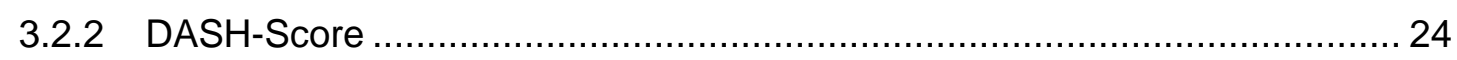

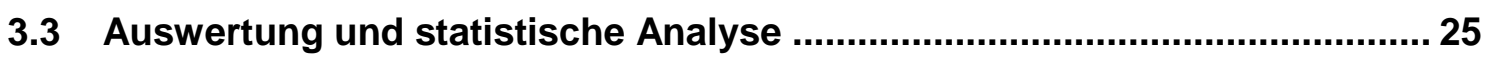

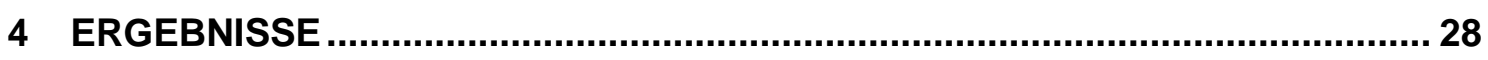

4.1 Deskriptive Darstellung der Stichprobe .......................................................... 28

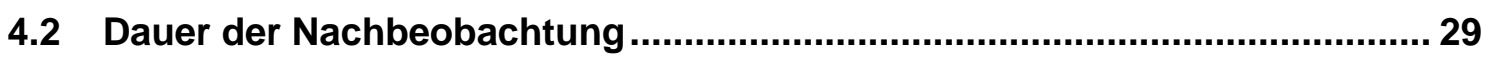

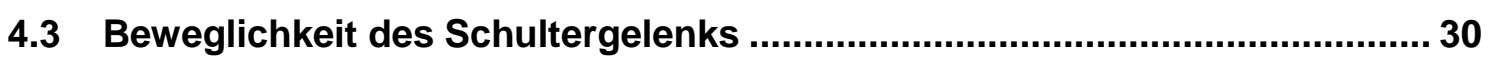

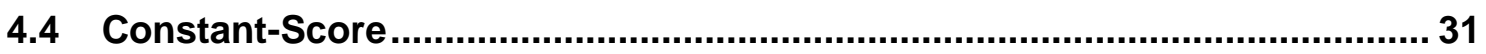

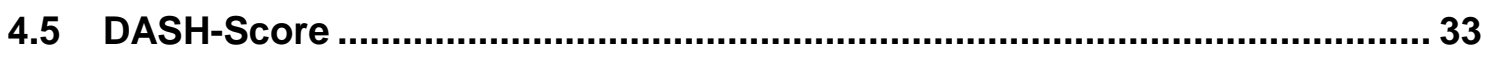

4.6 Einfluss des Geschlechts auf die Operationsergebnisse ............................ 35

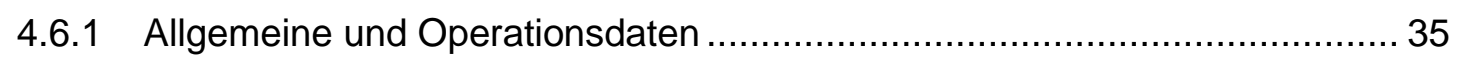

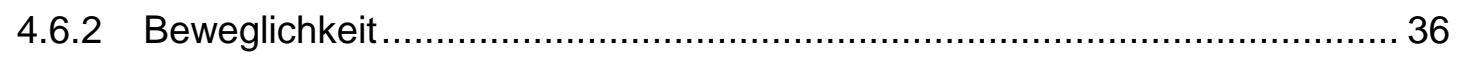

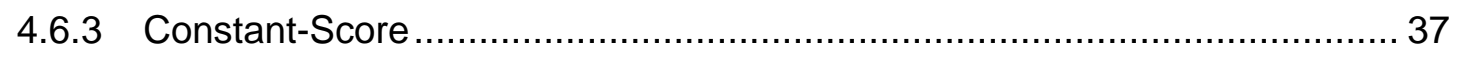

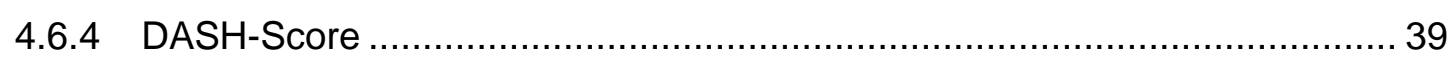


4.7 Einfluss des Alters auf die Operationsergebnisse ........................................ 40

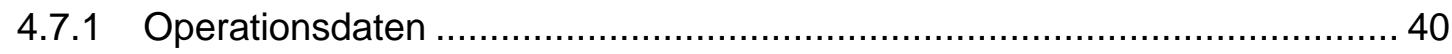

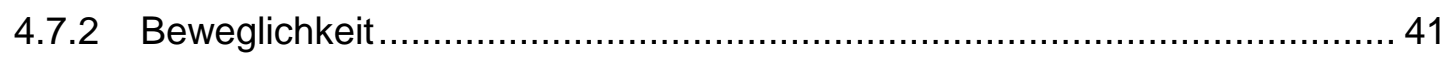

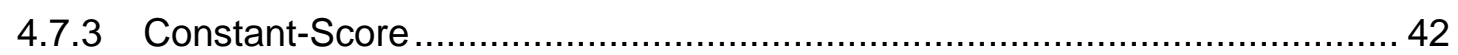

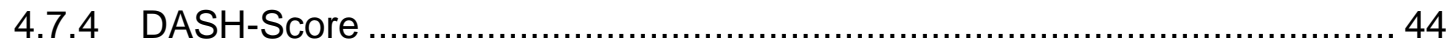

4.8 Radiologische Nachuntersuchung ................................................................ 45

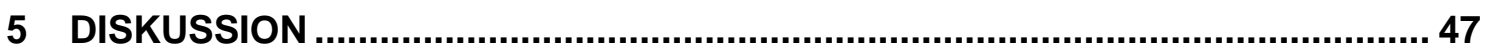

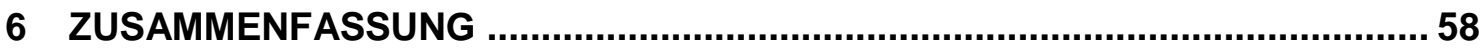

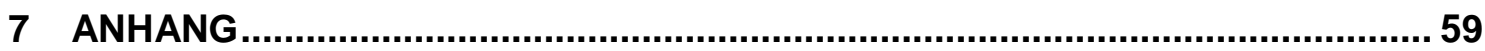

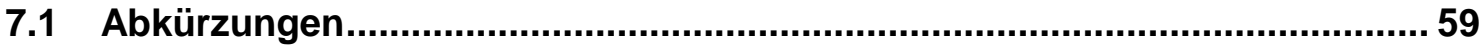

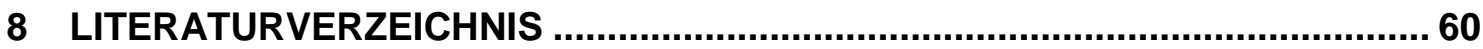

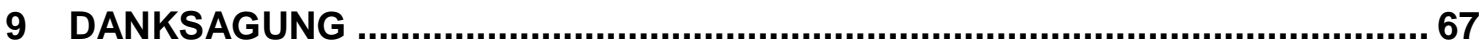

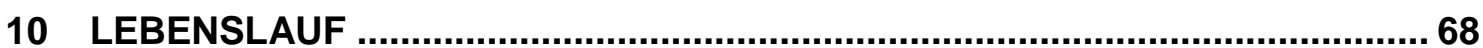




\section{$1 \quad$ Einleitung und Fragestellung}

Degenerative Veränderungen des Humeruskopfes können zahlreiche Ursachen haben. Am häufigsten kommen eine Omarthrose, eine rheumatoide Arthritis oder eine avaskuläre Humeruskopfnekrose vor. Die Folgen sind Schmerzen und - teilweise gravierende - Einschränkungen von Beweglichkeit und Funktion. Zur konservativen Behandlung werden physikalische Therapiemaßnahmen, die Anwendung topischer oder systemischer nichtsteroidaler Antiphlogistika bis hin zur intraartikulären Corticoid-Applikation eingesetzt. Bleibt die konservative Therapie erfolglos, werden chirurgische Maßnahmen in Form des Gelenkersatzes oder auch einer Arthrodese in Erwägung gezogen.

Außer den geschädigten Humeruskopf durch eine Endoprothese zu ersetzen, bemüht man sich in den letzten Jahren vermehrt um eine knochensparende, schonende, chirurgische Vorgehensweise und versucht, wenn möglich, lediglich die angegriffene Oberfläche des Humeruskopfes zu erneuern. Die zerstörten Bereiche werden abgetragen und die normale Anatomie mittels eines kappenförmigen Ersatzes wiederhergestellt. Besonders bei jüngeren, aktiven Patienten hält man sich auf diese Weise die Möglichkeit offen, zu einem späteren Zeitpunkt erforderlichenfalls eine Revision durchzuführen oder auf eine Totalendoprothese auszuweichen (Marquardt und Steinbeck, 2010). Bei älteren Patienten, die häufig weitere Erkrankungen aufweisen, wird besonders die vergleichsweise kurze Anästhesie- und Operationsdauer geschätzt (Mullett et al., 2007; Burgess et al., 2009; Raiss et al., 2009; Fakler et al., 2010). Im Vergleich mit der Totalendoprothetik sind als zusätzliche Vorteile des Oberflächenersatzes ein geringerer intraoperativer Blutverlust, ein geringeres Infektionsrisiko (Mullett et al., 2007; Fakler et al., 2010) sowie ein geringeres Risiko von Frakturen des Humerusschaftes (Boileau et al., 2006; Fakler et al., 2010) zu nennen.

Unter den unterschiedlichen Prothesenmodellen für den humeralen Oberflächenersatz sind besonders der zementfrei verankerte Copeland-Cup (Levy und Copeland, 2004; Levy et al., 2004; Mullett et al., 2007; Buchner et al., 2008; Scalise et al., 2008; Raiss et al., 2009; Raiss et al., 2010) sowie - in weit geringerem Umfang - die mit Zement zu verwendende ScanShoulder (Jonsson et al., 1988; Rydholm und Sjögren, 1993; Alund et al., 2000) untersucht worden.

Seit 2000 steht zusätzlich der ebenfalls zementierbare Durom Cup (Rüther, 2002) mit gegenüber der Scan-Shoulder verbesserten Verankerungsmöglichkeiten zur Verfügung. Da die Ergebnisse nach Verwendung des Durom-Cup bisher fast ausschließlich in Fallserien an Patienten mit einer rheumatoiden Arthritis untersucht wurden (Fink et al., 2004; Fürst et al., 2007, 2008; Fakler et al., 2010), ist es das Ziel der vorliegenden Arbeit, die funktionellen Re- 
sultate nach einem humeralen Oberflächenersatz durch einen Durom-Cup bei Patienten mit einer Omarthrose darzustellen und auszuwerten.

Im Einzelnen soll folgenden Fragen nachgegangen werden:

- Wie ändern sich Beweglichkeit und Schmerzen des erkrankten Schultergelenks nach dem Einsetzen eines Durom-Cup im Vergleich zum präoperativen Befund?

- Wie fallen die funktionellen Ergebnisse - gemessen mit dem Constant- und dem DASH-Score - aus?

- Werden die funktionellen Ergebnisse durch das Geschlecht oder Alter der Patienten beeinflusst?

- Wie häufig treten radiologisch erkennbare Lockerungszeichen des Oberflächenersatzes auf? 


\section{Literaturübersicht}

\subsection{Vorbemerkung zur Geschichte des Schultergelenksersatzes}

Vor Einführung von Alloarthroplastiken im Rahmen der Schulterchirurgie wurde als Maßnahme zur Erhaltung der Schulterfunktion bei einer tuberkulös-endzündlichen Gelenkzerstörung, nach Schussverletzungen oder Gelenkversteifungen die Resektion des Gelenkes vorgeschlagen. In der Literatur wird häufig der chirurgische Chirurg Charles White als Vorreiter der Schulterendoprothetik genannt: Da bei einem 14-jährigen Jungen nach einer Osteomyelitis das linke Schultergelenk versteift und schmerzhaft war, entschied er sich dafür, durch eine Humeruskopfresektion die Gelenkbeweglichkeit zumindest teilweise wiederherzustellen. Damit handelte er entgegen der seinerzeit üblichen Praxis, zerstörte Gelenke zu versteifen (Reimers, 1970). Weitere Chirurgen folgten diesem Beispiel und versuchten, die Gelenkmobilität durch Resektionsarthroplastiken zu erhalten (Reimers, 1970; Langenbeck von, 1974; Blauth und Donner, 1979).

Da nach zu ausgedehnter Resektion „Schlottergelenke“ beziehungsweise nach ungenügender Resektion Reankylosen auftraten, wurden Ende des 19./Anfang des 20. Jahrhundert Versuche unternommen, durch Interposition von Gewebe die postoperative Situation zu verbessern beziehungsweise den Operationserfolg auf längere Zeit auszudehnen. Als Interponate wurde autologes Gewebe in Form von Fett, Kapselmaterial, Muskel, Faszie, Hautabschnitte oder aus der Tibia oder Fibula des Patienten entnommene Knochenspäne verwendet (Payr, 1910; Murphy, 1913; Lexer, 1917; Baer, 1918; Brown et al., 1958). Mit Leichenmaterial kam auch heterologes Gewebe zum Einsatz (Lexer, 1908a, 1908b). Bedingt durch die Abstoßung der Transplantate, die Entstehung von Pseudarthrosen oder einer Inaktivitätsatrophie waren die Ergebnisse solcher Interpositionsarthroplastiken weniger zufriedenstellend und in der überwiegenden Anzahl der Fälle nicht lang anhaltend.

Erste Versuche zu einem anatomischen Ersatz des Schultergelenks wurden 1911 durch den deutschen Chirurgen König mit einer Nachbildung des proximalen Humerus aus Elfenbein unternommen (König, 1912, 1913). Im Jahr 1893 setzte der Franzose Pean eine Humeruskopfprothese aus einer Hartgummikugel ein. Die Kugel wurde von zwei Platinstreifen umschlossen, die an der Scapula beziehungsweise am Humerusschaft befestigt wurden (Pean, 1894). Solche frühen Versuche des künstlichen Gelenkersatzes scheiterten letztlich an noch nicht beherrschbaren Infektionen und Materialunverträglichkeiten. Erst in der zweiten Hälfte des 19. Jahrhunderts wurde nach positiven Erfahrungen auf dem Gebiet der Hüftendoprothetik auch den Schultergelenksarthroplastiken vermehrte Aufmerksamkeit gewidmet und man entwickelte spezielle Total- und Hemiarthroplastiken für dieses Gelenk. Als Wegbereiter der 
modernen Schulterendoprothetik gilt der Amerikaner Neer, der in den späten 1950er Jahren eine Vitallium-Prothese zur Behandlung von Humeruskopftrümmerfrakturen einsetzte und etwa 20 Jahre später, nach Erweiterung der Prothese um eine glenoidale Komponente, die ersten Totalalloplastiken zur Behandlung der Osteoarthritis durchführte (Neer, 1974, 2011).

\subsection{Formen und Einteilung der Schultergelenksendoprothesen}

Im Rahmen der Schultergelenksendoprothetik unterscheidet man heute prinzipiell zwischen Totalarthroplastiken, die sowohl den Humeruskopf als auch die Gelenkpfanne ersetzen, und den reinen Humeruskopfprothesen in Form von Hemiarthroplastiken. Grundsätzlich sind die Prothesen nach dem Prinzip der Kraft- oder Formschlüssigkeit aufgebaut. In der Mehrzahl der Fälle werden heute kraftschlüssige („unconstrained“) Hemiprothesen verwendet (Jerosch und Heisel, 2003). Die Indikationen für einen Schultergelenksersatz fassen Jerosch und Heisel (2003) wie folgt zusammen:

\section{Hemiarthroplastik}

- primäre degenerative Omarthrose mit konzentrischem Glenoid

- Humeruskopfnekrose mit noch intaktem Glenoid

- rheumatoide Arthritis mit schlechter Knochenqualität

- 4-Fragment-Fraktur des Humeruskopfes

- 3-Fragment-Fraktur des Humeruskopfes beim älteren Patienten

- Rotatorenmanschettendefekt-Arthropathie (optional: Delta-Prothese)

- proximaler Humerustumor ohne Gelenkbeteiligung

- sekundär dislozierte Osteosynthese

- schmerzhafte Pseudarthrose des proximalen Humerus mit Zerstörung des Humeruskopfes bei noch erhaltenem Glenoid

\section{Totalarthroplastik}

- primäre degenerative Omarthrose mit exzentrischem Abrieb der Fossa glenoidalis

○ rheumatoide Arthritis mit guter Knochenqualität

- postraumatische schmerzhafte Omarthrose mit Glenoidbeteiligung

- verhakte schmerzhafte Schultergelenkluxation nach dorsal oder ventral

- postinfektiöse schmerzhafte Omarthrose 


\subsection{Totalarthroplastiken}

Nach den grundsätzlichen Konstruktionsprinzipien ergeben sich bei den Totalarthroplastiken drei Untergruppen, die sich durch das Ausmaß der angestrebten Gelenkkongruenz und Stabilität und dem Grad der Verblockung unterscheiden. Die Gestaltung der Prothesen soll nicht nur knöcherne Zerstörungen des Schultergelenkes ausgleichen sondern gegebenenfalls auch die umgebende Weichteilstruktur rekonstruieren.

1. „unconstrained“ - Kraftschlüssige, ungekoppelte Systeme mit inkongruenten Kontaktflächen ermöglichen eine uneingeschränkte Beweglichkeit des Gelenks. Sie bieten durch den geringen Formschluss nur wenig Stabilität und erfordern daher eine intakte Schultergelenksmuskulatur und einen intakten Kapselbandapparat (Neer, 1974; Zhang et al., 2000; Buck et al., 2008; Neer, 2011).

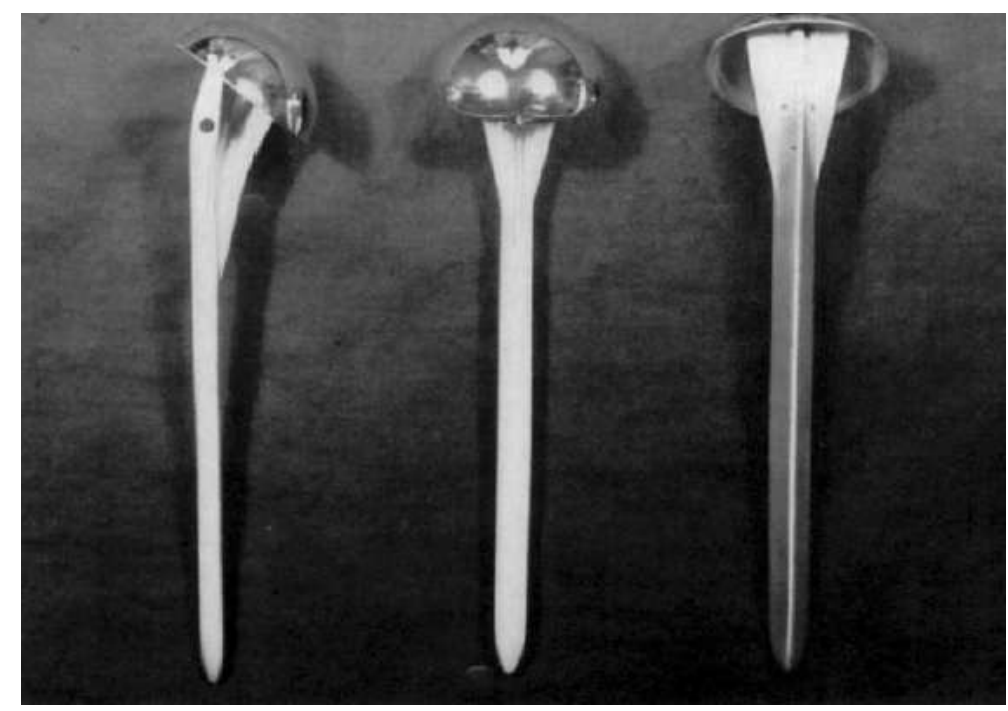

Abbildung 1: Klassische Neer I-Prothese als Beispiel für eine „unconstrained“-Prothese (Neer, 2011)

2. „semi-constrained“ - Halbgeführte, kraftschlüssige, ungekoppelte Systeme mit weitgehender Kongruenz der humeralen und glenoidalen Gelenkoberfläche reduzieren durch den erhöhten Formschluss die Gelenkbeweglichkeit. Modifizierungen dieses Konstruktionsprinzips werden bei vorgeschädigter Rotatorenmanschette gewählt, um die Stabilität zu erhöhen beziehungsweise das Luxationsrisiko zu verringern. An die Verankerung der stark beanspruchten glenoidalen Komponente werden bei diesem Prothesentyp 
hohe Anforderungen gestellt, um die Gefahr einer Implantatlockerung zu minimieren (McElwain und English, 1987; Nwakama et al., 2000).

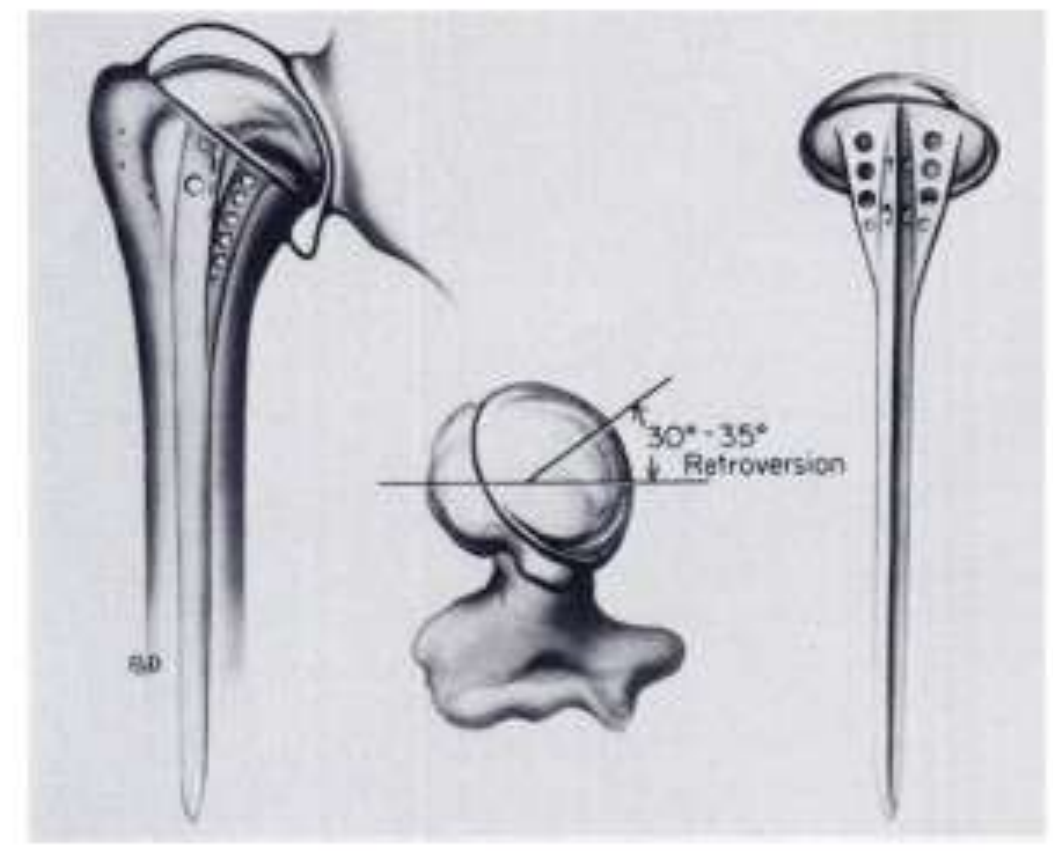

Abbildung 2: Klassische Neer II-Prothese als Beispiel für eine „semi-constrained“-Prothese (Neer, 1974)

Als Sonderform der semi-constrained-Prothese gilt die so genannte inverse Prothese, bei der eine Umkehr von Kopf und Pfanne realisiert wurde, um einen großen Bewegungsumfang zu garantieren.

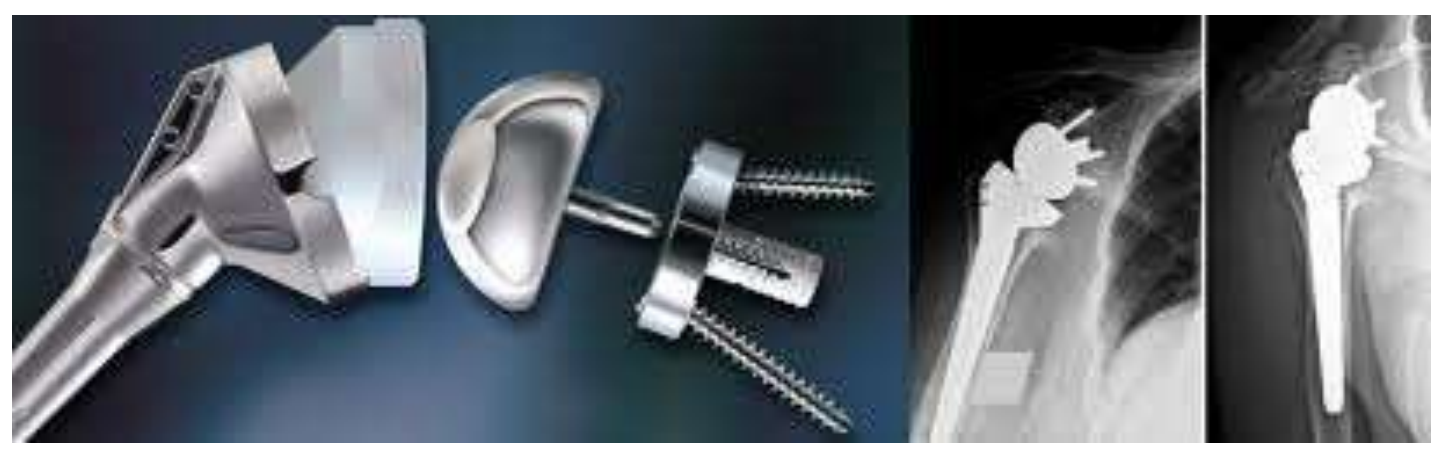

Abbildung 3: Inverse Schulterprothese nach Grammont (Delta-Schulterprothese, Fa. DePuy Orthopadics Deutschland, Kirkel-Limbach) 
3. „full-constrained“ - Formschlüssige, voll gekoppelte Systeme mit kongruenten Gelenkflächen sollen bei zerstörter Rotatorenmanschette die Stabilität des Gelenks gewährleisten, d.h. dass die Beweglichkeit noch weiter eingeschränkt wird und Rotation und Abduktion vom M. deltoideus übernommen werden müssen. Bei diesem Prothesentyp sind die Beanspruchungen der Pfannenverankerung und hieraus resultierend die Risiken einer Implantatlockerung noch höher (Reeves et al., 1972; Zippel, 1972; Kolbel und Friedebold, 1973, 1975; Cofield, 1979; Lettin, 1980; Post et al., 1980; Lettin et al., 1982; Wretenberg und Wallensten, 1999).

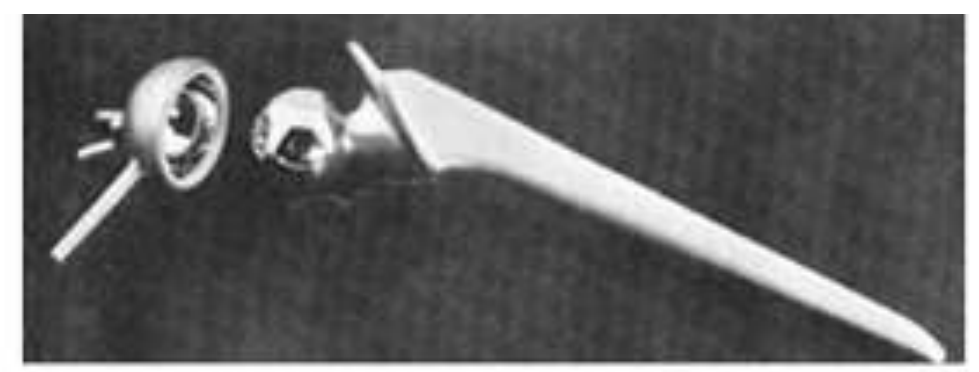

Abbildung 4: Stanmore-Prothese als Beispiel für eine „full-constrained“-Prothese (Lettin et al., 1982)

\subsection{Hemiarthroplastiken}

Unter Hemiarthroplastiken versteht man den alleinigen Ersatz des Humeruskopfes. Als Hemiarthroplastik entwickelte Prothesenmodelle werden allerdings gegebenenfalls auch mit einer glenoidalen Komponente kombiniert.

Nach dem historischen Entwicklungsstand wird zwischen Prothesen der ersten bis vierten Generation differenziert.

Als Prothesen der ersten Generation werden nichtmodulare Monoblockprothesen bezeichnet, bei denen Kopf und Schaft in einem nicht veränderbaren einteiligen Werkstück verbunden sind (Neer et al., 1982; Cofield, 1984; Barrett et al., 1987; Hawkins et al., 1989; Torchia et al., 1997). Sie gehen auf die ersten Prothesenmodelle von NEER zurück (Neer et al., 1982), und die Form des Humeruskopfes richtete sich nach der Kurvatur der glenoidalen Polyethylenkomponente im Rahmen der entsprechenden Totalendoprothese. 


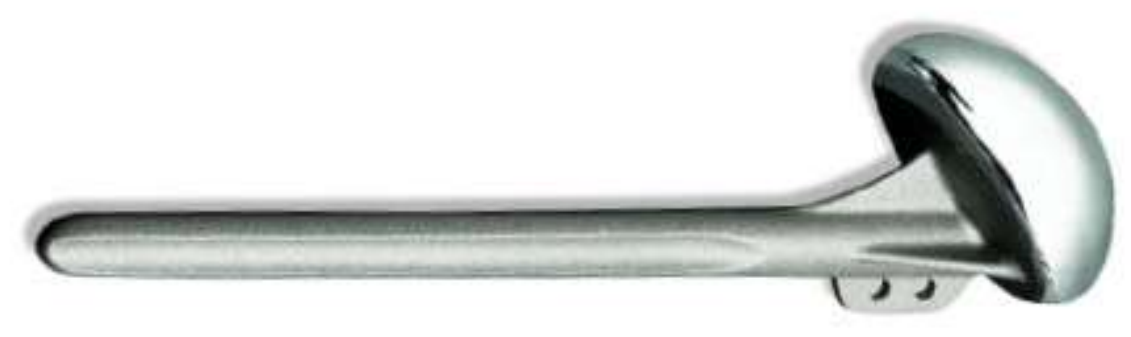

Abbildung 5: Monoblockprothese der ersten Generation (Neer 2-Prothese, SNKN-300 Sales Brochure fin, Fa. Smith \& Nephew, Memphis, Tennessee/USA)

Die Trennung von Kopf und Schaft ermöglicht bei Prothesen der zweiten Generation die Auswahl und Kombination verschiedener Humeruskopfgrößen und somit die Berücksichtigung individueller anatomischer Gegebenheiten (Fenlin et al., 1990; Fenlin et al., 1994; Gartsman et al., 1997; Godeneche et al., 2002). Dieses modulare Konzept erlaubt eine günstigere Anpassung an die Weichgewebe und berücksichtigt den lateralen humeralen Offset.

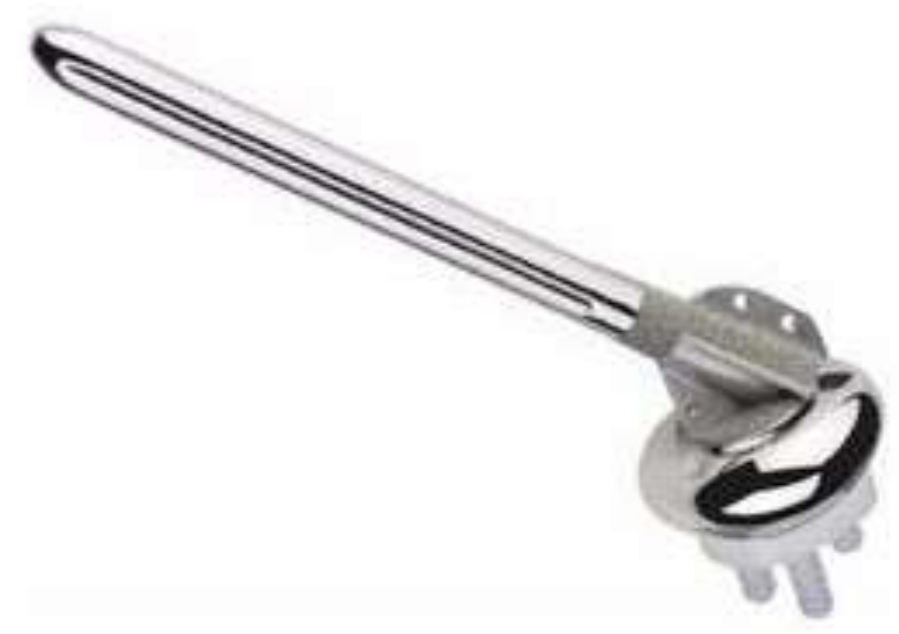

Abbildung 6: Prothese der zweiten Generation (Cofield 2-Prothese, SNKN-300 Sales Brochure fin, Fa. Smith \& Nephew, Memphis, Tennessee/USA)

Bei Prothesen der dritten Generation wurde erstmals der Schritt vollzogen, die Form des Prothesenkopfes den natürlichen Verhältnissen anzugleichen und nicht länger den Hume- 
ruskopf durch Fräsen an die Form der vorgebenen Prothese anpassen zu müssen. Als zusätzliche Formvariablen konnten nun mit Hilfe eines modularen Winkeladapters der Inklinationswinkel zwischen $125^{\circ}$ und $140^{\circ}$ vorgewählt und die Kopfkalotte exzentrisch verankert werden (Boileau und Walch, 1997; Walch und Boileau, 1999; Robertson et al., 2000).

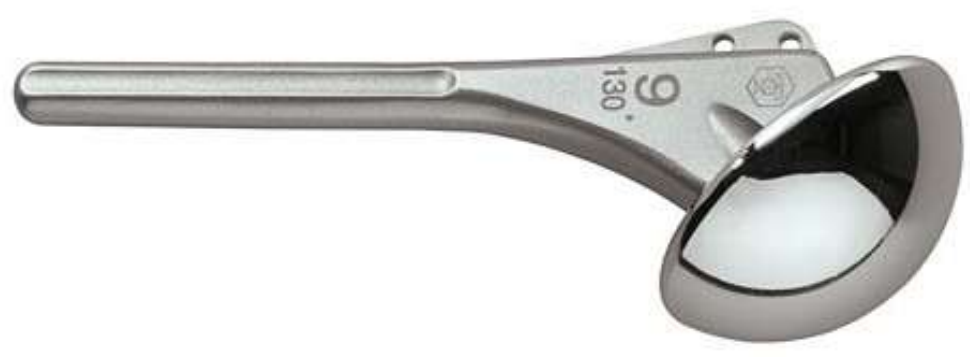

Abbildung 7: Prothese der dritten Generation (Aequalis-Prothese nach Walch und Boileau, Fa. Tornier, Amsterdam/Niederlande)

Prothesen der vierten Generation ermöglichen schließlich eine stufenfreie Anpassung der Komponenten um drei Achsen. Zu den Parametern Inklinationswinkel und Kopfexzentrizität kam eine Drehmöglichkeit im Kopf-Halsbereich der Prothese hinzu (Habermeyer und Ebert, 1999; Irlenbusch et al., 2011). „Die Schaftgeometrie wird von der Kopfposition unabhängig, der Schaft erlaubt die korrekte Positionierung der Kalotte auf der humeralen Resektionsfläche" (Habermeyer und Ebert, 1999). 


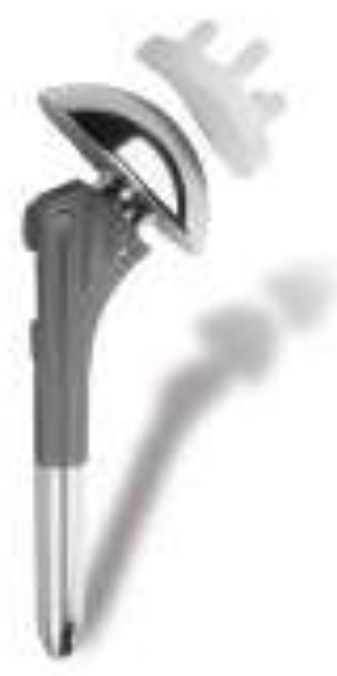

Abbildung 8: Prothese der vierten Generation (Anatomical Shoulder System, Fa. Zimmer Germany, Freiburg)

\subsection{Humeruskopf-Oberflächenersatz}

\subsubsection{Entwicklung und gebräuchliche Modelle}

Bei oberflächlichen Defekten des Humeruskopfes kann es ausreichend sein, nur die Oberfläche des Humeruskopfes zu ersetzen, um dem Patienten eine schmerzfreie Schulterfunktion zu ermöglichen. „Gedanklich leitet sich das Konzept zum Einen von der ResektionsInterpositions-Arthroplastik ab, indem als Interponat eine dünne Metallhaube gewählt wird. Zum Anderen kann man es als Miniaturisierung des gestielten Prothesentyps verstehen“ (Rüther, 2002).

Da als Voraussetzung für die erfolgreiche Verwendung eines Oberflächenersatzes ein umfänglicher und solider Erhalt des Humeruskopfes (Rüther, 2002) gilt, beschränken sich die Indikationen auf die Omarthrose (Levy und Copeland, 2001, 2004; Mullett et al., 2007; Buchner et al., 2008), die Osteoarthritis im Rahmen einer rheumatischen Arthritis (Fink et al., 2004; Fürst et al., 2007, 2008; Fakler et al., 2010) und die avaskuläre Humeruskopfnekrose (Levy und Copeland, 2001; Thomas et al., 2005; Mullett et al., 2007), aber auch Oberflächendefekten bei bestehenden Schäden der Rotatorenmanschette (Levy und Copeland, 2001; Thomas et al., 2005; Jerosch et al., 2008; Fakler et al., 2010). Kontraindikationen stellen Frakturen mit subkapitaler Komponente oder sehr ausgedehnte Humeruskopfnekrosen dar (Levy und Copeland, 2004; Raiss et al., 2009). 
Folgende Vorteile werden für den Oberflächenersatz des Humeruskopfes genannt:

- Als knochensparendes Verfahren wird der Oberflächenersatz bei jüngeren Patienten bevorzugt, da nach langjährigem Tragen der Prothese Wechseloperationen erleichtert werden (Burgess et al., 2009; Raiss et al., 2009)

- Individuelle Patientenparameter wie Inklination, posteriorer Offset und Retroversion lassen sich technisch vergleichsweise einfach und zuverlässig rekonstruieren (Levy und Copeland, 2004; Burgess et al., 2009; Raiss et al., 2009; Fakler et al., 2010).

- Besonders ältere Patienten profitieren von der kurzen Operations- und Anästhesiedauer, dem geringen intraoperativen Blutverlust und dem geringen Infektionsrisiko (Mullett et al., 2007; Fakler et al., 2010).

- Das Risiko einer periprothetischen Humerusschaftfraktur ist im Vergleich mit stilverankerten Prothesen geringer (Boileau et al., 2006; Fakler et al., 2010).

Als nachteilig gilt ein erschwerter Zugang zum Glenoid (Fakler et al., 2010).

Abbildung 9: Schematische Darstellung eines Oberflächenersatzes des Humeruskopfes in situ am Beispiel des Durom Shoulder cup (Fa. Zimmer, Freiburg) 
Zippel berichtete 1973, dass er bei einem Patienten mit rheumatoider Arthritis einen Oberflächenersatz des Humerus mit Hilfe einer einfachen Stahlkappe von $1 \mathrm{~mm}$ Wanddicke vorgenommen hatte. Die Kappe wurde über den Humeruskopf gestülpt und mit einer Schraube fest angepresst und fixiert. Der Patient war nach der Operation schmerzfrei und konnte seinen Arm gegenüber dem präoperativen Zustand besser bewegen (Zippel, 1973).

Zehn Jahre später verwendeten Steffee und Moore Femur-Oberflächencups für den Oberflächenersatz des Humerus (Steffee und Moore, 1984). Etwa zeitgleich entwickelte die Arbeitsgruppe Rydholm in Schweden einen Oberflächenersatz speziell für den Humeruskopf: Bei der Scan-Shoulder handelt es sich um eine halbkugelförmige Kappe aus Edelstahl mit einer Wandstärke von 1,7 mm. Der Cup besitzt an seiner Innenseite radiale und umlaufende Einbuchtungen, die die Haftung auf dem Humeruskopf und die Verdrängung überflüssigen Zements erleichtern sollen (Abbildung 10). Die Scan-Shoulder ist in 5 Durchmessern zwischen 30 und $46 \mathrm{~mm}$ erhältlich und wird in $135^{\circ}$ Inklination und $30^{\circ}$ Retroversion eingesetzt (Jonsson et al., 1986; Rydholm und Sjögren, 1993).

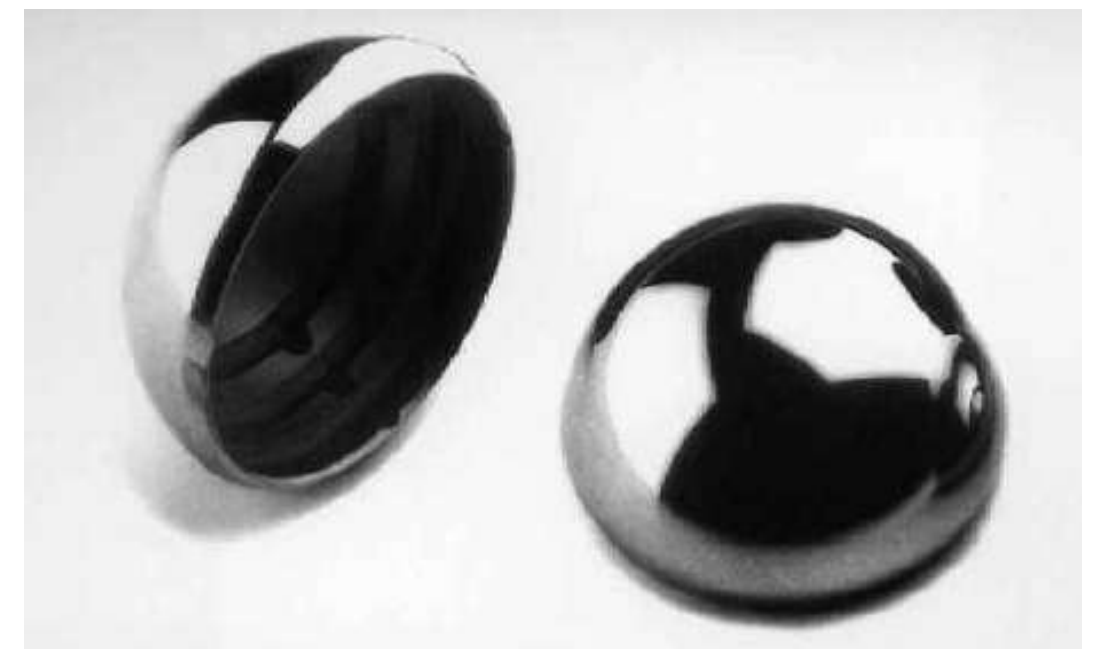

Abbildung 10: Scan-Shoulder (Fa. Scandimed, Sjöbo/Schweden; Alund et al., 2000)

Der sogenannte Copeland-Cup beruht auf der Totalendoprothese nach Copeland, deren humerale Komponente als Oberflächenersatz verwendet wurde. Die Kappe besteht aus einer Kobalt-Chrom-Molybdän-Legierung und besitzt einen zentralen, konischen Press-fit-Zapfen, der die Rotationsstabilität gewährleistet (Levy und Copeland, 2001). Aktuelle Modelle sind auf der Innenseite mit Hydoxxylapatit beschichtet, um das Einwachsverhalten zu fördern. Dadurch können Copeland-Cups zementfrei eingesetzt werden. Sie sind in verschiedenen 
Durchmessern, Kalottenhöhen sowie Formvarianten erhältlich, um die Kappen an das Ausmaß der Oberflächenzerstörung des Humerus inviduell anpassen zu können (Abbildung 11).

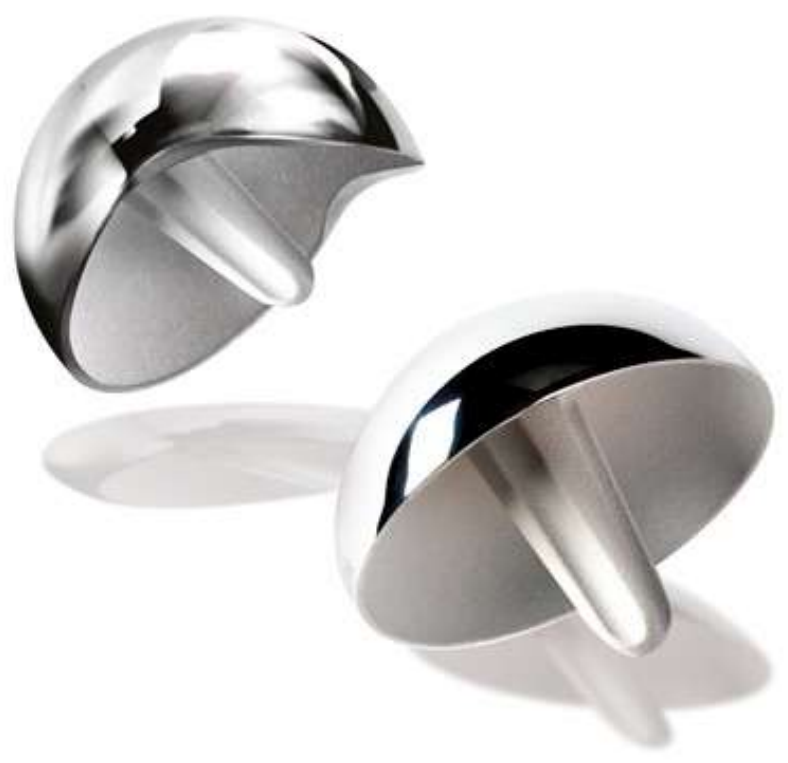

Abbildung 11: Copeland-Oberflächenersatz (Fa. Biomet Medical)

Der Durom Shoulder Cup besteht aus warmgeschmiedetem Protasul S-30 (FeCrNiMnMoNbN, ISO 5832-9), das eine hohe Korrosionsbeständigkeit aufweist. Die Form der Kappe ist den anatomischen Verhältnissen des Humeruskopfes nachgebildet. Sie wird in 7 verschiedenen Größen mit Durchmessern von $40 \mathrm{~mm}$ bis $52 \mathrm{~mm}$ angeboten. Im Inneren ist die Oberfläche des Cups grob gestrahlt, um eine gute Verbindung mit Knochenzement zu gewährleisten. Der Innenrand weist ein charakteristisches Relief aus hervortretenden bzw. eingebuchteten Bereichen auf, die einerseits der Zentrierung dienen und andererseits ein enges Anliegen am gefrästen Humeruskopf ermöglichen. Zentral findet sich ein zylindrischer Zapfen, der ebenfalls der Zentrierung dient und ein Abkippen der Prothese vom sphärisch gefrästen Humeruskopf verhindert. Eine zentrale Bohrung des Zapfens erlaubt eine geführte Implantation mittels Kirschnerdraht (Rüther, 2002). 


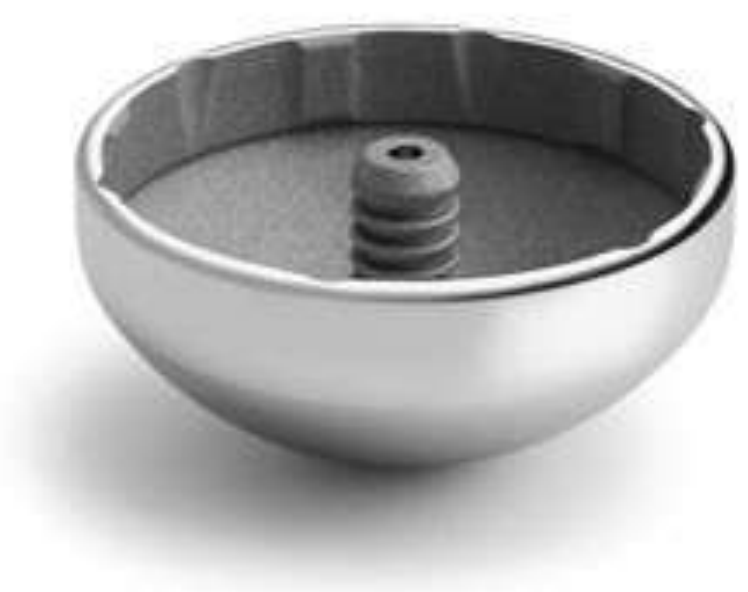

Abbildung 12: Durom® Shoulder Cup (Fa. Zimmer Germany, Freiburg)

Daneben exisitieren verschiedene weitere Kappenmodelle, die seltener verwendet werden und über die kaum klinische Studien existieren. An dieser Stelle sei wegen ihres besonderen Konstruktionsprinzips die EPOCA RH-Oberflächenersatzprothese (Fa. Synthes, Schweiz) genannt, die durch ihre mit Hydroxylapatit beschichtete, perforierte, leicht konisch geformte Innenkrone eine epiphysäre Fixation und eine gute primäre Press-fit-Stabilität erreichen soll (Marquardt et al., 2009).

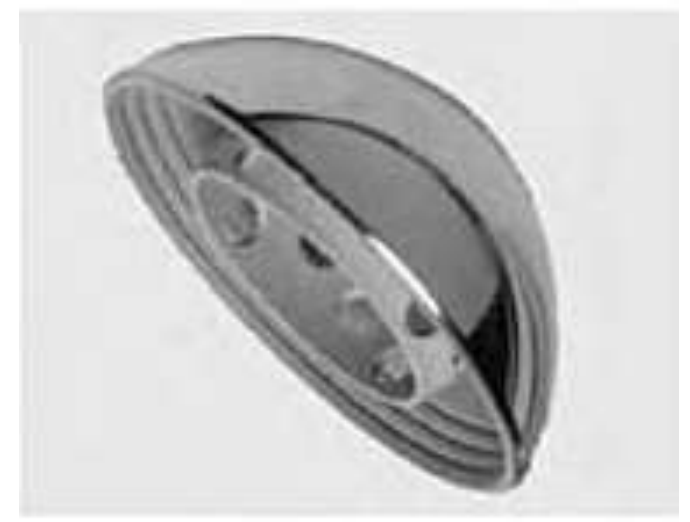

Abbildung 13: EPOCA RH-Oberflächenersatzprothese (Fa. Synthes, Schweiz; Marquardt et al., 2009)

Eine weitere Sonderform stellt das HemiCAP-System dar (Fa. Arthrosurface, Franklin, Massachussetts/USA). Die Oberflächenkappe aus Chrom-Kobalt-Legierung ist in verschiedenen 
Größe erhältlich und lässt sich homogen in die gesunde Gelenkoberfläche einfügen. Sie ist mit einem Konus und einer Titanplasmaschicht versehen und wird auf einen separaten, zylindrischen Schraubenkörper aufgeschraubt.

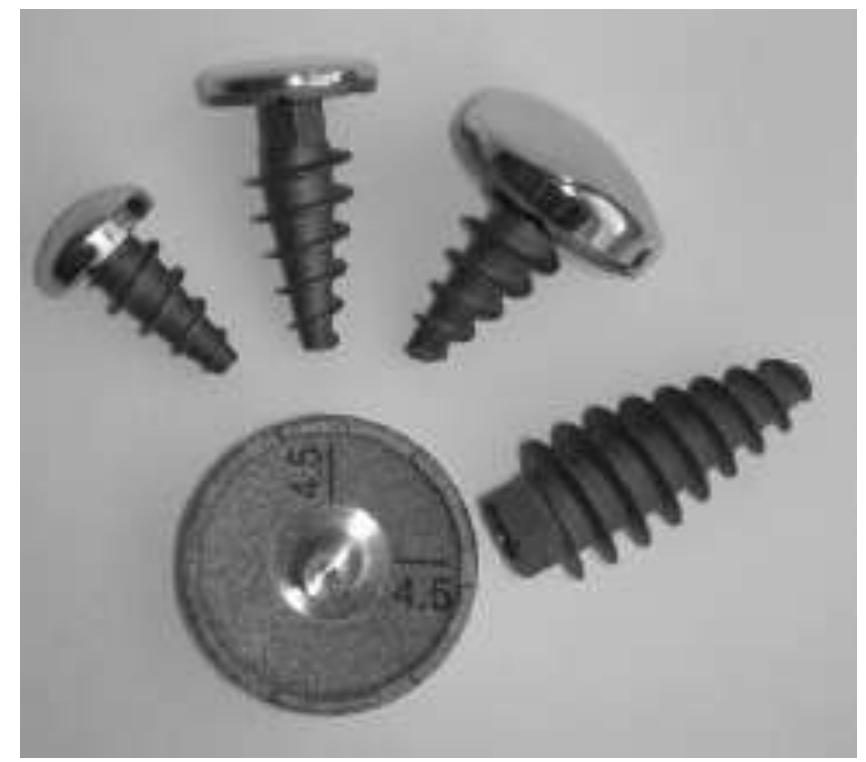

Abbildung 14: HemiCAP-Shoulder Resurfacing System (Fa. Arthrosurface, Franklin, Massachussetts/USA)

\subsubsection{Studienlage}

In der folgenden Tabelle 1 sind die Kenndaten der vorliegenden klinischen Studien zu den aktuell gebräuchlichen, humeralen Oberflächenersatzprothesen zusammengefasst. Auf die funktionellen Ergebnisse der einzelnen Studien wird im Rahmen der Diskussion im Vergleich mit den eigenen Resultaten eingegangen.

Man sieht in der Übersicht, dass sich bislang erst wenige Studien mit jeweils meist recht kleinen Fallzahlen mit den Ergebnissen nach Oberflächenersatz des Humeruskopfes befasst haben. Als am besten untersucht, stellt sich der Copeland-Cup dar, über den acht Studien (hiervon zwei mit identischem Patientengut [Raiss et al., 2009, Raiss et al., 2010]) mit Nachuntersuchungsergebnissen von insgesamt 189 Schultergelenken publiziert wurden. Die Studien umfassen das gesamte Indikationsspektrum des Oberflächenersatzes und Patienten jeden Alters. Durch die Möglichkeit, den Copeland-Cup mit einer entsprechenden glenoidalen Komponente zu einer Totalendoprothese kombinieren zu können, wurden häufig Vergleiche zwischen dem Oberflächenersatz und einer (kontralateralen) Totalendoprothese vorge- 
nommen. Als Besonderheit führten Buchner et al. (2008) eine prospektive Matched-PairStudie zum direkten Vergleich zwischen den Ergebnissen nach Einsetzen eines CopelandCups oder einer Aequalis-TEP bei primärer Osteoarthritis durch. Die Patienten wurden nach Alter, Geschlecht und Diagnose gepaart. Hierbei erwies sich der Copeland Oberflächenersatz nach einem Jahr der TEP als nur geringfügig unterlegen, so dass die Autoren eine Verwendung der Cups empfehlen.

Im Gegensatz zum Copeland-Cup werden die Scan-Shoulder und der Durom-Cup zementiert. Zur Scan-Shoulder liegen lediglich drei Studien über insgesamt 116 Schultergelenke vor. Als einzige Indikation wurde die rheumatoide Arthritis gewählt. Die jüngste Studie (Alund et al., 2000) kommt wegen des häufigen Auftretens radiologischer Lockerungszeichen zu dem Schluss, dass ein routinemäßiger Einsatz der Scan-Shoulder bei rheumatoider Arthritis trotz zufriedenstellender klinischer Ergebnisse nach vier Jahren zumindest in Frage gestellt werden muss.

Als alternativen zementierbaren Oberflächenersatz entwickelte Rüther den Durom Cup, der zur Verbesserung der Verankerung neben einem Innenrelief aus hervorstehenden und eingebuchteten Bereichen einen zentral liegenden Zapfen, eine grob gestrahlte Oberfläche und einen zylindrischen Abschluss am Äquator besitzt (Fürst et al., 2008). Erste kurz- und mittelfristige Ergebnisse wurden in vier Fallserien - davon zwei mit identischem Patientengut (Fürst et al., 2007, 2008) - publiziert. Insgesamt wurden in die Studien 180 Schultergelenke eingeschlossen, die in der überwiegenden Mehrzahl der Fälle durch eine rheumatoide Arthritis geschädigt waren.

Die vorliegende Untersuchung soll eine Lücke schließen, und die funktionellen Ergebnisse nach Einsetzen eines Durom Cup bei Patienten mit einer Omarthrose analysieren. 
Tabelle 1: $\quad$ Übersicht über klinische Studiem zum humeralen Oberflächenersatz mit verschiedenen Kappenmodellen

$(\mathrm{OP}=$ Operationen, Pat. = Patienten, SG = Schultergelenke, Mon. = Monate, NU = Nachuntersuchung, OA = Omarthrose, RA = rheumatoide Arthritis, HKN = Humeruskopfnekrose, RM = Defekte der Rotatorenmanschette, PA = posttraumatische Arthrose, IN = Instabilität, k.A. = keine Angabe, TEP = Totalendoprothese)

\begin{tabular}{|c|c|c|c|c|c|c|c|c|}
\hline Autoren & $\begin{array}{l}\text { Anzahl } \\
\text { OP } \\
\text { Pat./SG } \\
\end{array}$ & $\begin{array}{c}\text { Alter } \\
\text { (Jahre) }\end{array}$ & $\begin{array}{l}\text { Follow } \\
\text { up } \\
\text { (Jahre) }\end{array}$ & $\begin{array}{c}\text { Anzahl } \\
\text { NU } \\
\text { Pat./SG }\end{array}$ & $\begin{array}{l}\text { Indika- } \\
\text { tionen }\end{array}$ & Prothesenart & Endpunkte & $\begin{array}{l}\text { Studien- } \\
\text { typ }\end{array}$ \\
\hline $\begin{array}{l}\text { Levy und } \\
\text { Copeland, } \\
2001\end{array}$ & $94 / 103$ & 64,3 & 6,8 & $\begin{array}{l}\text { 1. } 68 \mathrm{SG} \\
\text { 2. } 35 \mathrm{SG}\end{array}$ & $\begin{array}{l}\text { OA, RA, } \\
\text { RM, HKN, } \\
\text { IN }\end{array}$ & $\begin{array}{l}\text { 1. Copeland-TEP } \\
\text { 2. Copeland-Cup }\end{array}$ & Constant-Score & Fallserie \\
\hline $\begin{array}{l}\text { Levy und } \\
\text { Copeland, } \\
2004\end{array}$ & $\begin{array}{l}\text { 1. } 42 / 43 \\
\text { 2. } 37 / 46\end{array}$ & $\begin{array}{l}\text { 1. } 71,5 \\
\text { 2. } 73,4\end{array}$ & $\begin{array}{l}\text { 1. } 7,6 \\
\text { 2. } 4,4\end{array}$ & $\begin{array}{l}\text { k.A./39 } \\
\text { k.A./30 }\end{array}$ & $\mathrm{OA}$ & $\begin{array}{l}\text { 1. Copeland-TEP } \\
\text { 2. Copeland-Cup }\end{array}$ & Constant-Score & Fallserie \\
\hline $\begin{array}{l}\text { Levy et al., } \\
2004\end{array}$ & $62 / 75$ & 60,7 & 6,5 & $62 / 75$ & RA & $\begin{array}{l}\text { 1. Copeland-TEP } \\
(\mathrm{n}=33 \mathrm{~S} .) \\
\text { 2. Copeland-Cup } \\
(\mathrm{n}=42 \mathrm{~S} .)\end{array}$ & $\begin{array}{l}\text { Constant-Score } \\
\text { Patientenzufriedenheit }\end{array}$ & Fallserie \\
\hline $\begin{array}{l}\text { Thomas et al., } \\
2005\end{array}$ & $44 / 48$ & 70 & 2,8 & $44 / 48$ & OA, RA & Copeland-Cup & Constant-Score & Fallserie \\
\hline $\begin{array}{l}\text { Mullett et al., } \\
2007\end{array}$ & $29 / 29$ & 84,3 & 4,7 & $21 / 21$ & $\begin{array}{l}\mathrm{OA}, \mathrm{RA}, \\
\mathrm{RM}, \mathrm{HKN}\end{array}$ & $\begin{array}{l}\text { 1. Copeland-TEP } \\
\text { 2. Copeland-Cup }\end{array}$ & $\begin{array}{l}\text { Constant-Score } \\
\text { Patientenzufriedenheit }\end{array}$ & Fallserie \\
\hline $\begin{array}{l}\text { Buchner et al., } \\
2008\end{array}$ & $\begin{array}{l}\text { 1. } 22 / 22 \\
\text { 2. } 22 / 22\end{array}$ & $\begin{array}{l}\text { 1. } 61,4 \\
\text { 2. } 61,1\end{array}$ & 1 & $\begin{array}{l}\text { 1. } 22 / 22 \\
\text { 2. } 22 / 22\end{array}$ & $\mathrm{OA}$ & $\begin{array}{l}\text { 1. Copeland-Cup } \\
\text { 2. Aequalis-TEP }\end{array}$ & Constant-Score & $\begin{array}{l}\text { Prospektive } \\
\text { vergleichende } \\
\text { Studie }\end{array}$ \\
\hline $\begin{array}{l}\text { Jerosch et al., } \\
2008\end{array}$ & $25 / 25$ & 69 & 2,2 & $25 / 25$ & $\mathrm{RM}$ & Copeland-Cup & $\begin{array}{l}\text { Constant-Score } \\
\text { Patientenzufriedenheit }\end{array}$ & Fallserie \\
\hline
\end{tabular}




\begin{tabular}{|c|c|c|c|c|c|c|c|c|}
\hline Autoren & $\begin{array}{c}\text { Anzahl } \\
\text { OP } \\
\text { Pat./SG }\end{array}$ & $\begin{array}{c}\text { Alter } \\
\text { (Jahre) }\end{array}$ & $\begin{array}{l}\text { Follow } \\
\text { up } \\
\text { (Jahre) }\end{array}$ & $\begin{array}{c}\text { Anzahl } \\
\text { NU } \\
\text { Pat./SG }\end{array}$ & $\begin{array}{l}\text { Indika- } \\
\text { tionen }\end{array}$ & Prothesenart & Endpunkte & $\begin{array}{l}\text { Studien- } \\
\text { typ }\end{array}$ \\
\hline $\begin{array}{l}\text { Scalise et al., } \\
2008\end{array}$ & $\begin{array}{l}\text { 1. } 16 / 16 \\
\text { 2. } 76 / 76 \\
\text { 3. } 62 / 62\end{array}$ & $\begin{array}{l}45 \\
67 \\
60\end{array}$ & $\begin{array}{l}\text { 1. } 1,6 \\
\text { 2. k.A. } \\
\text { 3. } 0,8\end{array}$ & $\begin{array}{l}\text { 1. } 16 / 16 \\
\text { 2. } 76 / 76 \\
\text { 3. } 62 / 62\end{array}$ & $\begin{array}{l}\text { OA, RA, } \\
\text { HKN, PA }\end{array}$ & $\begin{array}{l}\text { 1. Copeland- oder } \\
\text { DePuy-Cup } \\
\text { 2. TEP } \\
\text { 3. HemiCAP }\end{array}$ & $\begin{array}{l}\text { Schmerz, Funktion, } \\
\text { Zufriedenheit }\end{array}$ & Fallserie \\
\hline $\begin{array}{l}\text { Raiss et al., } \\
\text { 2009/Raiss et } \\
\text { al., } 2010\end{array}$ & $13 / 17$ & 48 & 3 & $14 / 17$ & HKN & $\begin{array}{l}\text { 1. Copeland- oder } \\
\text { EPOCA-RH Cup }\end{array}$ & Constant-Score & Fallserie \\
\hline $\begin{array}{l}\text { Jonsson et al., } \\
1988\end{array}$ & 8 & 37,4 & $\begin{array}{l}1.2 \\
2.10\end{array}$ & $\begin{array}{l}1.5 \\
2.5\end{array}$ & RA & $\begin{array}{l}\text { 1. (Scan?)-Cup } \\
\text { 2. Arthrodese }\end{array}$ & $\begin{array}{l}\text { UCLA-Score (Kraft, } \\
\text { Schmerz, Funktion, Be- } \\
\text { weglichkeit) }\end{array}$ & $\begin{array}{l}\text { Vergleichende } \\
\text { Studie }\end{array}$ \\
\hline $\begin{array}{l}\text { Rydholm und } \\
\text { Sjögren, } 1993\end{array}$ & $70 / 84$ & 53 & 4,2 & $59 / 72$ & RA & Scan-Cup & $\begin{array}{l}\text { Schmerz, Funktion, Be- } \\
\text { weglichkeit, Patienten- } \\
\text { zufriedenheit }\end{array}$ & Fallserie \\
\hline $\begin{array}{l}\text { Alund et al., } \\
2000\end{array}$ & $41 / 47$ & 55 & 4,4 & $33 / 39$ & RA & Scan-Shoulder & $\begin{array}{l}\text { Constant-Score } \\
\text { Patientenzufriedenheit }\end{array}$ & Fallserie \\
\hline $\begin{array}{l}\text { Fink et al., } \\
2004\end{array}$ & $39 / 45$ & 62,7 & 3,8 & $39 / 45$ & RA & Durom Cup & Constant-Score & Fallserie \\
\hline $\begin{array}{l}\text { Fürst et al., } \\
2007 / \text { Fürst et } \\
\text { al., } 2008\end{array}$ & $35 / 42$ & 61,4 & 6,1 & $29 / 35$ & RA & Durom Cup & Constant-Score & Fallserie \\
\hline
\end{tabular}




\begin{tabular}{|l|c|c|c|c|c|l|l|l|}
\hline \multicolumn{1}{|c|}{ Autoren } & $\begin{array}{c}\text { Anzahl } \\
\text { OP } \\
\text { Pat./SG }\end{array}$ & $\begin{array}{c}\text { Alter } \\
\text { (Jahre) }\end{array}$ & $\begin{array}{c}\text { Follow } \\
\text { up } \\
\text { (Jahre) }\end{array}$ & $\begin{array}{c}\text { Anzahl } \\
\text { NU } \\
\text { Pat./SG }\end{array}$ & $\begin{array}{c}\text { Indika- } \\
\text { tionen }\end{array}$ & \multicolumn{1}{|c|}{ Prothesenart } & \multicolumn{1}{|c|}{$\begin{array}{c}\text { Endpunkte } \\
\text { typ }\end{array}$} \\
\hline $\begin{array}{l}\text { Fakler et al., } \\
2010\end{array}$ & $49 / 52$ & 70,7 & 3,4 & $38 / 39$ & $\begin{array}{l}\text { OA, RA, } \\
\text { RM, HKN, } \\
\text { PA }\end{array}$ & Durom Cup & $\begin{array}{l}\text { Constant-Score } \\
\text { DASH-Score }\end{array}$ & Fallserie \\
\hline
\end{tabular}




\section{$3 \quad$ Material und Methodik}

\subsection{Patienten}

In der orthopädischen Abteilung des St. Vincenz-Hospitals in Brakel werden für die Rekonstruktion des Schultergelenks indikationsbezogen zahlreiche Varianten des Gelenkersatzes verwendet wie beispielsweise inverse Schulterprothesen (Delta Xtend), Traumaprothesen (Affinis Mathysmedical), anatomische Prothesen (Biomet) und vereinzelt als zementfreie Oberflächenersatzvarianten Copeland-Cup und Epoca-Cup sowie Biomet TESS-Prothesen. Der Durom-Cup kam nur bei erhaltener Rotatorenmanschette zum Einsatz.

In die Studie wurden zunächst 31 Patienten eingeschlossen, bei denen in den Jahren 2001 bis 2010 ein Oberflächenersatz des Schultergelenks mit Hilfe einer Durom-CupProthese (Fa. Zimmer Germany, Freiburg) vorgenommen wurde. Zwei Patienten mussten nachträglich ausgeschlossen werden: Eine Patientin verstarb im März 2009 an einer Herzinsuffizienz. Und bei einer Patientin erfolgte zwei Jahre nach Implantation der Durom-Cup-Prothese eine Reoperation mit Wechsel auf eine Delta-InverseProthese.

Es verblieben 29 Patienten für die vorliegenden Untersuchung. Hiervon waren drei Patienten beidseitig operiert worden, so dass 32 Schultergelenke zur Auswertung gelangten.

Die Untersuchungen wurden zwischen Anfang November 2009 und Ende März 2010 durchgeführt.

\subsection{Datenerhebung}

Aus den Krankenakten wurden Anamnese und die präoperativen Untersuchungsbefunde, einschließlich des präoperativen Bewegungsumfanges, entnommen.

Bei allen Patienten wurde der postoperative Bewegungsumfang der Schulter in Abduktion, Flexion und Außenrotation ermittelt. 
Bei 26 der 29 Patienten konnten Röntgenaufnahmen im Rahmen des Nachbehandlungsschemas (6 Monate postoperativ, danach jährlich bzw. ab 7 Jahre postoperativ alle zwei Jahre). Bezüglich der Durchführung und Verwendung der Röntgenaufnahmen lag ein positives Votum der Ethikkommission vor.

Zur Beurteilung der Schulterfunktionsfähigkeit wurden der Constant-Score (Constant und Murley, 1987) und die Parameter „subjektive Beurteilung“, „Erwartungshaltung Schmerz" und „Erwartungshaltung Funktion“ des DASH-Scores (Hudak et al., 1996) verwendet. 


\subsubsection{Constant-Score}

In den Constant-Score fließen vier Parameter ein:

- Beurteilung der Schmerzen (15 Punkte),

- Fähigkeit zur Verrichtung von Alltagsaktivitäten (20 Punkte),

- Beweglichkeit (40 Punkte),

- Kraft (25 Punkte).

Maximal sind 100 Punkte erreichbar.

Beim Constant-Score wird der Bewegungsumfang mit maximal 40 Punkten bewertet. Der Bewegungsumfang wird aktiv ermittelt und entsprechend der möglichen Bewegungsebenen des Schultergelenkes in Flexion (maximal 10 Punkte), Abduktion (maximal 10 Punkte), Außenrotation (maximal 10 Punkte) und Innenrotation (maximal 10 Punkte) untersucht.

Die Flexion und Abduktion werden mittels Goniometer am stehenden Patienten ermittelt und die Gradzahlen nach dem in Tabelle 2 dargestellten Schema in Punktwerte umgerechnet. Die Bestimmung des Ausmaßes der Außenrotation wird anhand der Stellung von Hand und Ellenbogen definiert. Die im Weiteren aufgezählten Teilkomponenten werden jeweils mit zwei Punkten bewertet, wenn sie der Patient ausführen kann. Die Bestimmung des Ausmaßes der Innenrotation richtet sich nach der Höhe auf der Körperrückseite, die mit dem Handrücken der zu untersuchenden oberen Extremität erreicht werden kann. 
Tabelle 2: Beurteilung der Schulterfunktion nach dem Constant-Score

\begin{tabular}{|c|c|c|c|}
\hline Parameter & \multicolumn{2}{|l|}{ Kategorie } & Punkte \\
\hline \multirow[t]{4}{*}{ Schmerzen } & \multicolumn{2}{|l|}{ keine } & 15 \\
\hline & \multicolumn{2}{|l|}{ leichte } & 10 \\
\hline & \multicolumn{2}{|l|}{ mäßige } & 5 \\
\hline & \multicolumn{2}{|l|}{ schwere } & 0 \\
\hline \multirow[t]{3}{*}{ Aktivität } & \multirow{3}{*}{\multicolumn{2}{|c|}{$\begin{array}{l}\text { uneingeschränkte Arbeitsfähigkeit } \\
\text { uneingeschränkte Sportfähigkeit } \\
\text { uneingeschränkte Nachtruhe }\end{array}$}} & 4 \\
\hline & & & 4 \\
\hline & & & 2 \\
\hline \multirow[t]{6}{*}{ Beweglichkeit } & \multirow[t]{6}{*}{ Abduktion } & $151-180^{\circ}$ & 10 \\
\hline & & $121-150^{\circ}$ & 8 \\
\hline & & $91-120^{\circ}$ & 6 \\
\hline & & $61-90^{\circ}$ & 4 \\
\hline & & $31-60^{\circ}$ & 2 \\
\hline & & bis $30^{\circ}$ & 0 \\
\hline \multirow[t]{6}{*}{ Beweglichkeit } & \multirow[t]{6}{*}{ Flexion } & $151-180^{\circ}$ & 10 \\
\hline & & $121-150^{\circ}$ & 8 \\
\hline & & $91-120^{\circ}$ & 6 \\
\hline & & $61-90^{\circ}$ & 4 \\
\hline & & $31-60^{\circ}$ & 2 \\
\hline & & bis $30^{\circ}$ & 0 \\
\hline \multirow[t]{13}{*}{ Beweglichkeit } & \multirow{6}{*}{\multicolumn{2}{|c|}{$\begin{array}{l}\text { Außenrotation } \\
\text { Hand hinter dem Kopf - Ellbogen nach vorne } \\
\text { Hand hinter dem Kopf - Ellbogen nach hinten } \\
\text { Hand auf dem Kopf - Ellbogen nach vorne } \\
\text { Hand auf dem Kopf - Ellbogen nach hinten } \\
\text { volle Elevation vom Kopf aus }\end{array}$}} & \\
\hline & & & 2 \\
\hline & & & 2 \\
\hline & & & 2 \\
\hline & & & 2 \\
\hline & & & 2 \\
\hline & \multirow{7}{*}{\multicolumn{2}{|c|}{$\begin{array}{l}\text { Innenrotation: Handrücken auf Höhe } \\
\text { - } \\
\text { des 7. Brustwirbelkörpers } \\
\text { - } \\
\text { des 12. Brustwirbelkörpers } \\
\text { des lumbosakralen Übergangs } \\
\text { - } \\
\text { des lliosakralgelenks } \\
\text { der Glutäen } \\
\text { des Trochanter major }\end{array}$}} & \\
\hline & & & 10 \\
\hline & & & 8 \\
\hline & & & 6 \\
\hline & & & 4 \\
\hline & & & 2 \\
\hline & & & 0 \\
\hline
\end{tabular}




\subsubsection{DASH-Score}

Mit Hilfe des DASH-Scores (Disabilities of the Arm, Shoulder and Hand, Hudak et al., 1996) können Patienten subjektiv das Operationsergebnis und die Erfüllung ihrer Erwartungen hinsichtlich Schmerzen und Funktion mit Hilfe eines Fragebogens aus 78 Items erfassen. Für jede Einzelfrage sind 5 Antwortmöglichkeiten vorgegeben, wobei für die jeweils beste Möglichkeit 1 Punkt und die jeweils schlechteste 5 Punkte vergegeben werden.

Darüber hinaus kann eine modifizierte - auch hier eingesetzte - Kurzfassung verwendet werden, mit deren Hilfe subjektive Beurteilung und die Erwartungshaltung Schmerzen sowie Funktion nach dem gleichen Muster beurteilt werden (Tabelle 3), und die eine gute Einsicht in die Zufriedenheit der Patienten mit inrem Operationsergebnis erlaubt.

Tabelle 3: $\quad$ Modifizierter DASH-Score

\begin{tabular}{|l|l|c|}
\hline Parameter & Kategorie & Punkte \\
\hline \multirow{2}{*}{ Subjektive Beurteilung } & sehr gut & 1 \\
& gut & 2 \\
& befriedigend & 3 \\
& ausreichend & 4 \\
& mangelhaft & 5 \\
\hline \multirow{2}{*}{ Erwartungshaltung Schmerzen } & sehr viel besser & 1 \\
& besser als erwartet & 2 \\
& so wie erwartet & 3 \\
& schlechter als erwartet & 4 \\
& sehr viel schlechter als erwartet & 5 \\
\hline \multirow{2}{*}{ Erwartungshaltung Funktion } & sehr viel besser & 1 \\
& besser als erwartet & 2 \\
& so wie erwartet & 3 \\
& schlechter als erwartet & 4 \\
& sehr viel schlechter als erwartet & 5 \\
\hline
\end{tabular}




\subsection{Auswertung und statistische Analyse}

In die Auswertung wurden folgende Variablen einbezogen:

\begin{tabular}{|l|}
\hline Geschlecht \\
\hline Alter \\
\hline Alter in Kategorien \\
\hline operierte Seite \\
\hline Voroperation an der Schulter \\
\hline Jahr der OP \\
\hline Indikation \\
\hline CS Schmerz präOP \\
\hline CS Schmerz postOP \\
\hline CS Schmerz Differenz post-präOP \\
\hline CS Aktivität präOP \\
\hline CS Aktivität postOP \\
\hline CS Aktivität Differenz post-präOP \\
\hline CS Beweglichkeit präOP \\
\hline CS Beweglichkeit postOP \\
\hline CS Beweglichkeit Differenz post-präOP \\
\hline
\end{tabular}

\begin{tabular}{|l|}
\hline CS Kraft präOP \\
\hline CS Kraft postOP \\
\hline CS Kraft Differenz post-präOP \\
\hline CS präOP \\
\hline CS postOP \\
\hline CS Differenz post-prä \\
\hline Subjektive Beurteilung \\
\hline Erwartungshaltung Schmerzen \\
\hline Erwartungshaltung Funktion \\
\hline Abduktion präOP \\
\hline Flexion präOP \\
\hline Außenrotation präOP \\
\hline Abduktion postOP \\
\hline Flexion postOP \\
\hline Außenrotation postOP \\
\hline
\end{tabular}

[CS = Constant Score, OP = Operation/operativ $]$

Die Auswertungen wurden mit dem Programmpaket STATISTICA (Fa. StatSoft Inc., Tulsa/USA) durchgeführt.

Für stetige Variablen ${ }^{1}$ wurden als statistische Kenngrößen der arithmetische Mittelwert, die Standardabweichung, Median sowie Minimum und Maximum ermittelt. Für diskrete Variablen ${ }^{2}$ wurden die absoluten und relativen Häufigkeiten (in Prozent) angegeben.

1 Die Abstände der aufeinander folgenden Intervalle der Variablen sind konstant (hier zum Beispiel Lebensjahre).

2 Die Ergebnisse sind in Kategorien angeordnet (hier zum Beispiel Geschlecht männlich oder weiblich). 
Es wurden folgende statistische Tests verwendet (Sachs, 2004; Pospeschill, 2006):

- Unterschiede in den Mittelwerten stetiger Variablen bei abhängigen Untersuchungen $^{3}$ (hier zum Beispiel Constant-Score bei einem Patienten post- versus präoperativ) wurden mit Hilfe des Wilcoxon-Tests für Paardifferenzen analysiert. Als Ergebnis wird eine Tabelle angegeben, die neben der Anzahl gültiger Wertepaare (Gültige N), einige Zwischenergebnisse der Signifikanzberechnung (T, Z) und die Irrtumswahrscheinlichkeit $p$ enthält.

- Zur Ermittlung von Unterschieden in den Mittelwerten stetiger Variablen bei unabhängigen 4 Untersuchungen (hier zum Beispiel mittleres Lebensalter bei Frauen und Männern) wurde der U-Test von Mann und Whitney verwendet.

- Zur Untersuchung der Unterschiede in der Häufigkeitsverteilung diskreter Variablen wurde der Chi²-Test gewählt. Sind zwei Stichproben A und B (hier zum Beispiel Frauen/Männer) nach zwei Ausprägungen eines Merkmals M1 oder M2 aufgeteilt (hier zum Beispiel Alter bis/über 68 Jahre), wird eine Vierfeldertafel mit den Besetzungszahlen a, b, c und d aufgestellt:

\begin{tabular}{|c|c|c|c|}
\hline & \multicolumn{2}{|c|}{ Stichprobe } & \\
\hline Merkmal & A & B & \\
\hline M1 & a & c & S1 $=\mathrm{a}+\mathrm{c}$ \\
\hline M2 & b & d & S2 $=$ b+d \\
\hline & S3 $=\mathrm{a}+\mathrm{b}$ & $\mathrm{S} 4=\mathrm{c}+\mathrm{d}$ & \\
\hline
\end{tabular}

Mit dem $\chi^{2}$-Test für die Auswertung von Vierfeldertafeln wird geprüft, ob sich die Randsummen S1 bis S4 hinsichtlich des untersuchten Merkmals unterscheiden oder nicht. Eine Verallgemeinerung auf mehrere Stichproben und/oder mehrere Merkmale bietet die Darstellung in einer $\mathrm{k}^{*} \mathrm{c}$ - felder-Tafel, die ebenfalls mit Hilfe des $\chi^{2}$-Tests auf statistisch signifikante Unterschiede hinsichtlich der Merkmalsausprägungen untersucht werden kann.

3 Untersuchungen am gleichen Patienten (z.B. Befund vor und nach einer Behandlung am gleichen Patienten)

$4 \quad$ Untersuchungen an verschiedenen Patienten (z.B. Vergleich der Befunde von Männern und Frauen) 
Bei kleinen Besetzungszahlen lieferte der exakte Test nach Fisher zuverlässige-

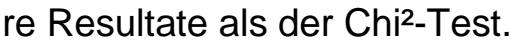

Ergebnis eines jeden Tests ist die Irrtumswahrscheinlichkeit p. Je kleiner $p$, desto gröBer ist die Wahrscheinlichkeit, dass ein postulierter Unterschied oder Zusammenhang zwischen Stichproben tatsächlich existiert. Als Schwelle für ein statistisch signifikantes Ergebnis wurde eine Irrtumswahrscheinlich von $p \leq 0,05$ gewählt. Für den Fall $p>0,05$ wurde das Ergebnis als nicht signifikant angegeben: Ein Unterschied oder Zusammenhang war nicht mit ausreichender Sicherheit nachzuweisen. 


\section{$4 \quad$ Ergebnisse}

\subsection{Deskriptive Darstellung der Stichprobe}

In die Untersuchung wurden 29 Patienten einbezogen. Es handelte sich um 16 Frauen $(55,2 \%)$ und 13 Männer (44,8\%). Das mittlere Alter der Patienten betrug 70,2 $\pm 7,1$ Jahre bei einer Spannweite der Werte von 55 bis 83 Jahren. Zwölf Patienten (41,4\% waren bis 68 Jahre alt und 17 Patienten (58,6\%) älter als 68 Jahre.

Bei drei Patienten wurden beide Schultergelenke operiert, so dass insgesamt 32 Schultergelenke zur Auswertung vorlagen. Bezogen auf die Schultergelenke betrug das mittlere Alter 70,6 \pm 6,9 Jahre und 19 Schultergelenke $(59,4 \%)$ stammten von weiblichen, 13 Schultergelenke $(40,6 \%)$ von männlichen Patienten.

Bei der Einteilung der Patienten in Alterskategorien stammten 12 Schultern (37,5\%) von Patienten bis 68 Jahre und 20 Schultern (62,5\%) von Patienten über 68 Jahre.

Es wurden 15 linke $(46,8 \%)$ und 17 rechte $(53,2 \%)$ Schultergelenke operiert.

Aus der Verteilung der Operationen wird ersichtlich, dass der Oberflächenersatz des Humerus mit dem Durom Shoulder Cup bis einschließlich zum Jahr 2006 nur vereinzelt durchgeführt wurde. Ab 2007 betrug die jährliche Operationsfrequenz fünf bis neun Fälle jährlich (Abbildung 15). 


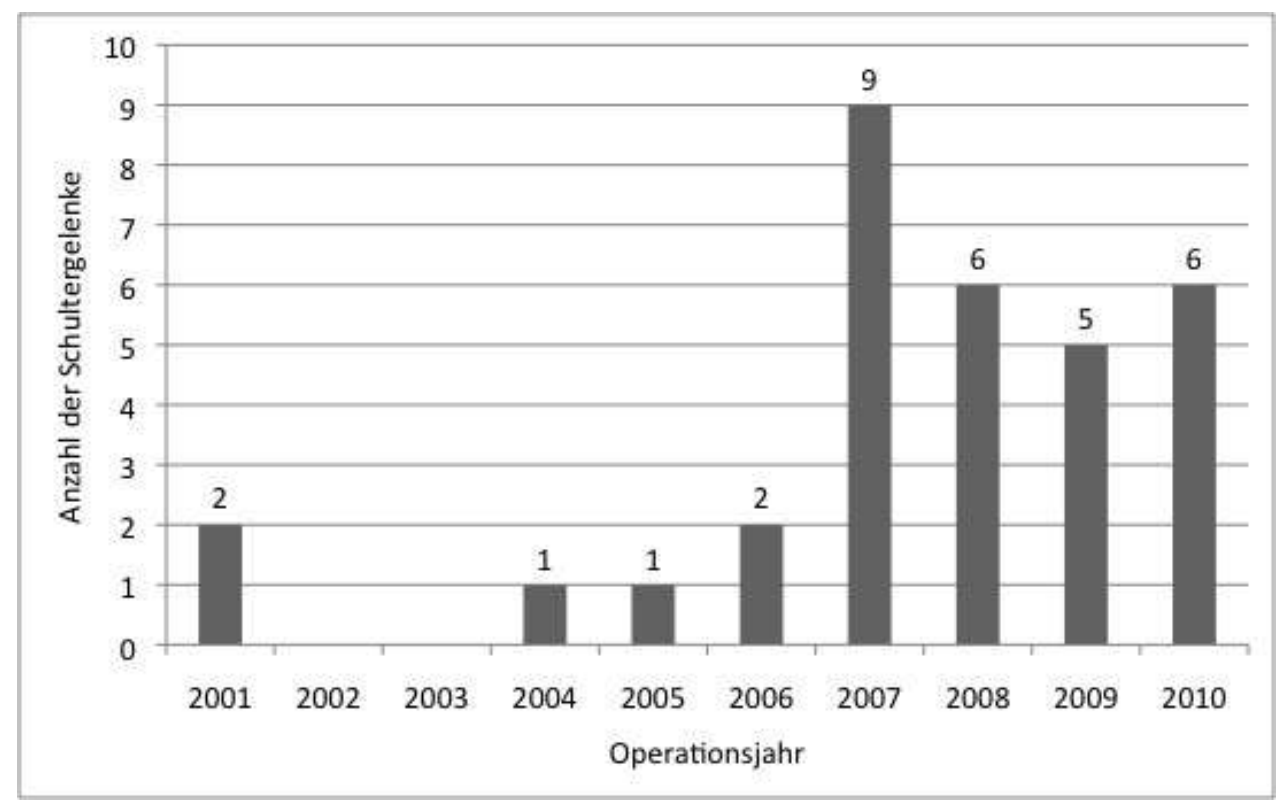

Abbildung 15: Zeitliche Verteilung der Operationen $(n=32$ Schultergelenke)

Bei fünf Schultergelenken (15,6\%) waren Voroperationen vorgenommen worden. Es hatte sich ausnahmslos um eine arthroskopische subacromiale Dekompression (SAD) auf Grund einer Omarthrose gehandelt. Der Abstand zwischen Voroperation und dem aktuellen Oberflächenersatz betrug zweimal 1 Jahr, zweimal 2 Jahre und einmal 10 Jahre.

Als Operationsindikation für den Oberflächenersatz des Humeruskopfes war in der überwiegenden Anzahl der Fälle, bei 30 Schultergelenken (93,8\%) eine primäre Omarthrose genannt worden. Bei einem Schultergelenk $(3,1 \%)$ lag eine sekundäre posttraumatische Omarthrose und bei bei einem weiteren Schultergelenk $(3,1 \%)$ eine sekundäre Omarthrose als Folge einer Humeruskopfnekrose vor.

\subsection{Dauer der Nachbeobachtung}

Die Nachuntersuchungen fanden zwischen 0,5 und 9 Jahren nach dem Oberflächenersatz des Humeruskopfes statt. Die durchschnittliche Nachbeobachtungsdauer betrug $2,7 \pm 2,1$ Jahre. 


\subsection{Beweglichkeit des Schultergelenks}

Postoperativ hatten sich sowohl Abduktion als auch Flexion und Außenrotation statistisch hoch signifikant verändert (Tabelle 4). Der Ausmaß der Abduktion besserte sich von präoperativ $69,1 \pm 29,7^{\circ}$ auf postoperativ $118,8 \pm 21,4^{\circ}$, die Flexion von $81,6 \pm 31,1^{\circ}$ auf $123,1 \pm 24,2^{\circ}$ und die Außenrotation von $23,0 \pm 16,2^{\circ}$ auf $42,7 \pm 13,6^{\circ}$.

Tabelle 4: Verbesserungen der Beweglichkeit nach dem Oberflächenersatz des Schultergelenks $(n=32)$

\begin{tabular}{|l|c|c|c|c|}
\hline Parameter & präoperativ & postoperativ & Differenz & $\mathrm{p}$ \\
\hline Abduktion $\left({ }^{\circ}\right)$ & $69,1 \pm 29,7$ & $118,8 \pm 21,4$ & $49,7 \pm 25,8$ & $<0,0001$ \\
\hline & & & & \\
Flexion $\left({ }^{\circ}\right)$ & $81,6 \pm 31,1$ & $123,1 \pm 24,2$ & $41,5 \pm 28,2$ & $<0,0001$ \\
\hline & & & & \\
Außenrotation $\left(^{\circ}\right)$ & $23,0 \pm 16,2$ & $42,7 \pm 13,6$ & $19,7 \pm 15,3$ & $<0,0001$ \\
\hline
\end{tabular}

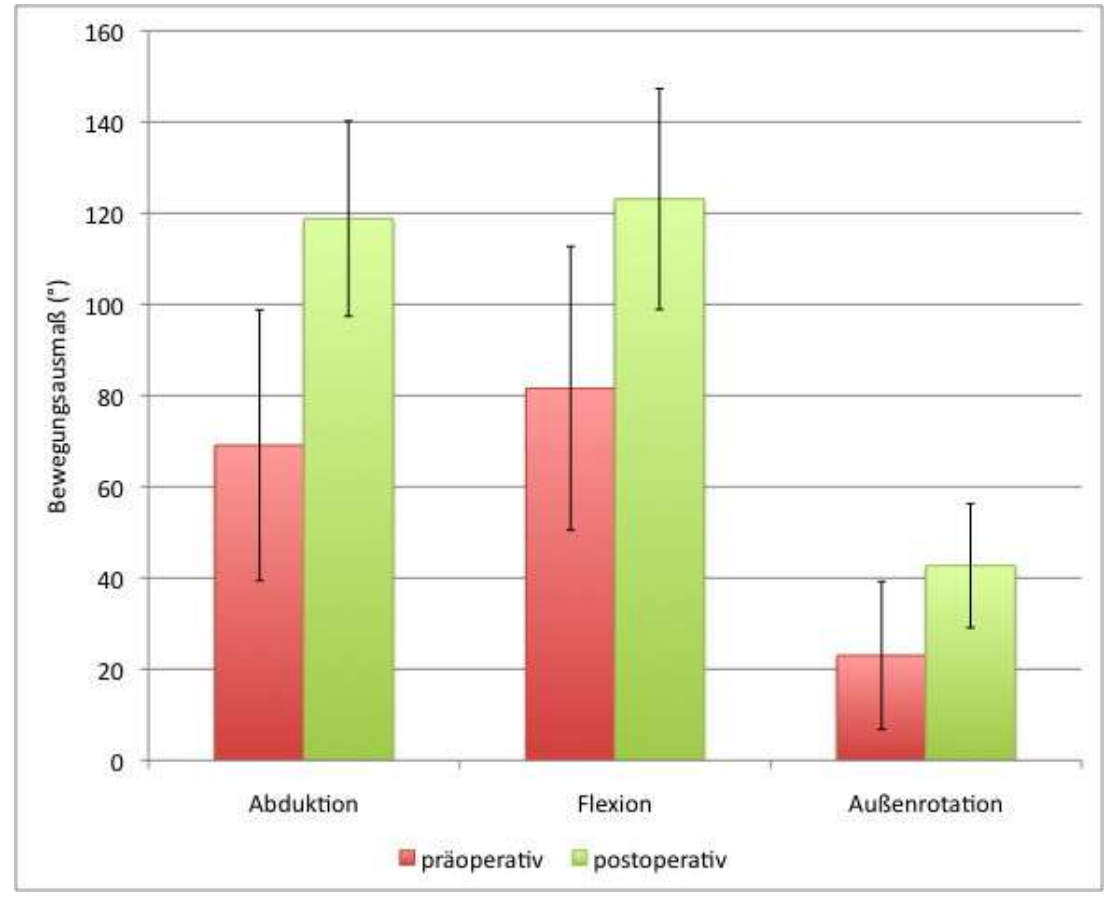

Abbildung 16: Verbesserungen der Beweglichkeit nach dem Oberflächenersatz des Schultergelenks $(n=32)$ 


\subsection{Constant-Score}

Der Constant-Score hatte vor der Operation im Durchschnitt 44,5 \pm 10,6 Punkte betragen und sich postoperativ statistisch hoch signifikant auf 61,4 \pm 13,1 Punkte verbessert (Abbildung 17).

Auch bezüglich der einzelnen Kategorien des Constant-Scores waren statistisch hoch signifikante Verbesserungen bei jedem Parameter zu verzeichnen (Tabelle 5).

Tabelle 5: Veränderungen der Einzelparameter des Constant-Scores sowie des Gesamtscores bei 32 Schultergelenken (Angabe in Punkten; MW \pm SD)

\begin{tabular}{|l|c|c|c|c|}
\hline Parameter & präoperativ & postoperativ & Differenz & $p$ \\
\hline Schmerzen & $7,3 \pm 2,1$ & $10,2 \pm 2,2$ & $2,9 \pm 2,1$ & $<0,0001$ \\
\hline Aktivität & $8,8 \pm 2,7$ & $12,3 \pm 2,8$ & $3,5 \pm 3,0$ & $<0,0001$ \\
\hline Beweglichkeit & $15,7 \pm 5,9$ & $24,0 \pm 6,4$ & $8,3 \pm 5,4$ & $<0,0001$ \\
\hline Kraft & $12,9 \pm 3,1$ & $15,0 \pm 4,1$ & $2,1 \pm 3,1$ & $<0,001$ \\
\hline & & & & $<0,0001$ \\
\hline
\end{tabular}




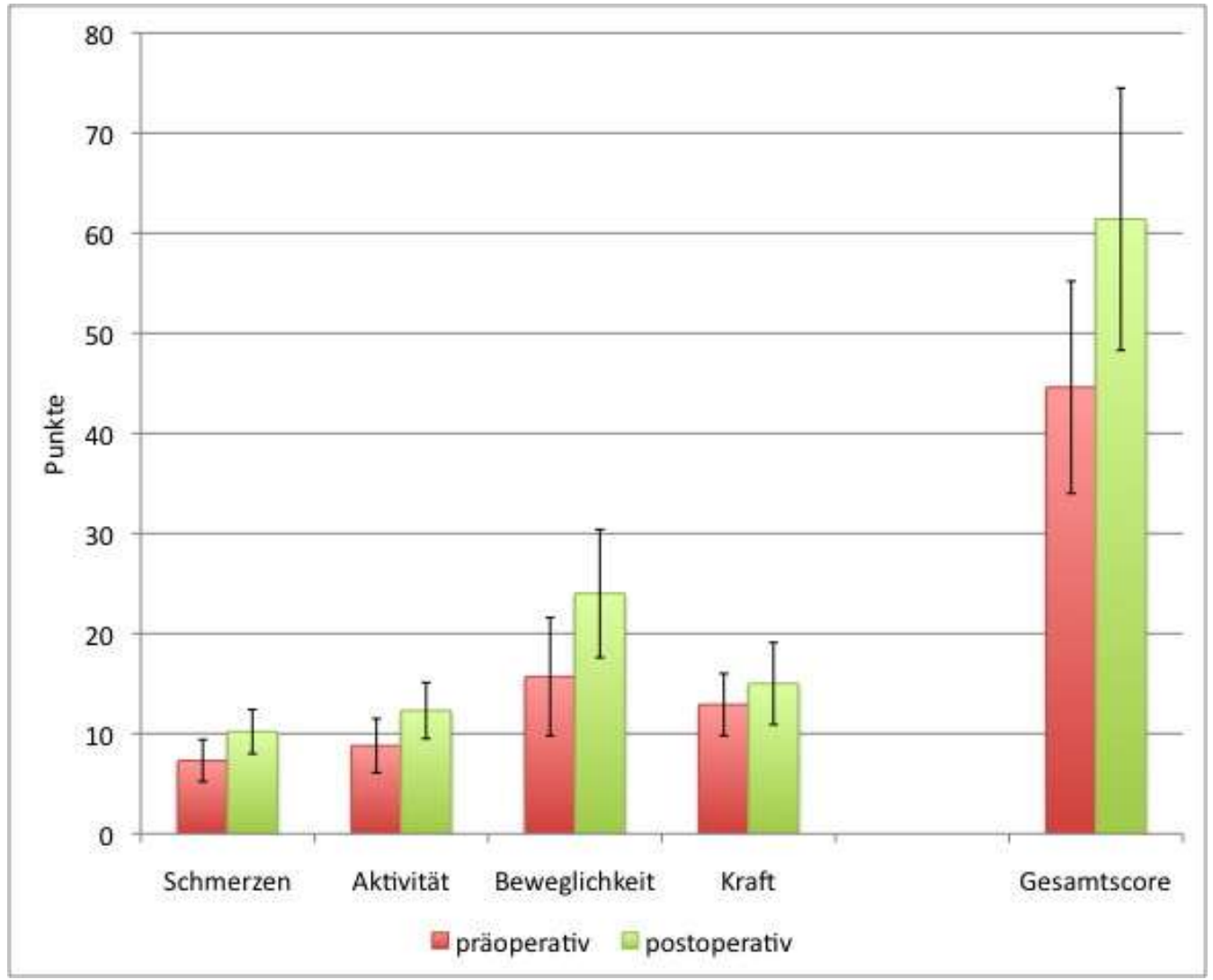

Abbildung 17: Veränderungen der Einzelparameter des Constant-Scores sowie des Gesamtscores bei 32 Schultergelenken $(\mathrm{MW} \pm \mathrm{SD})$ 


\subsection{DASH-Score}

Sieben Patienten (21,9\%) beurteilten ihr Operationsergebnis als sehr gut und 14 $(43,8 \%)$ als gut. Weitere 7 Patienten $(21,9 \%)$ erachteten das Ergebnis als befriedigend und die restlichen 4 Patienten (12,5\%) fanden es noch ausreichend. Kein Patient vergab die Beurteilung mangelhaft.

Die durchschnittliche Punktzahl betrug 2,3 \pm 1,0 Punkte.

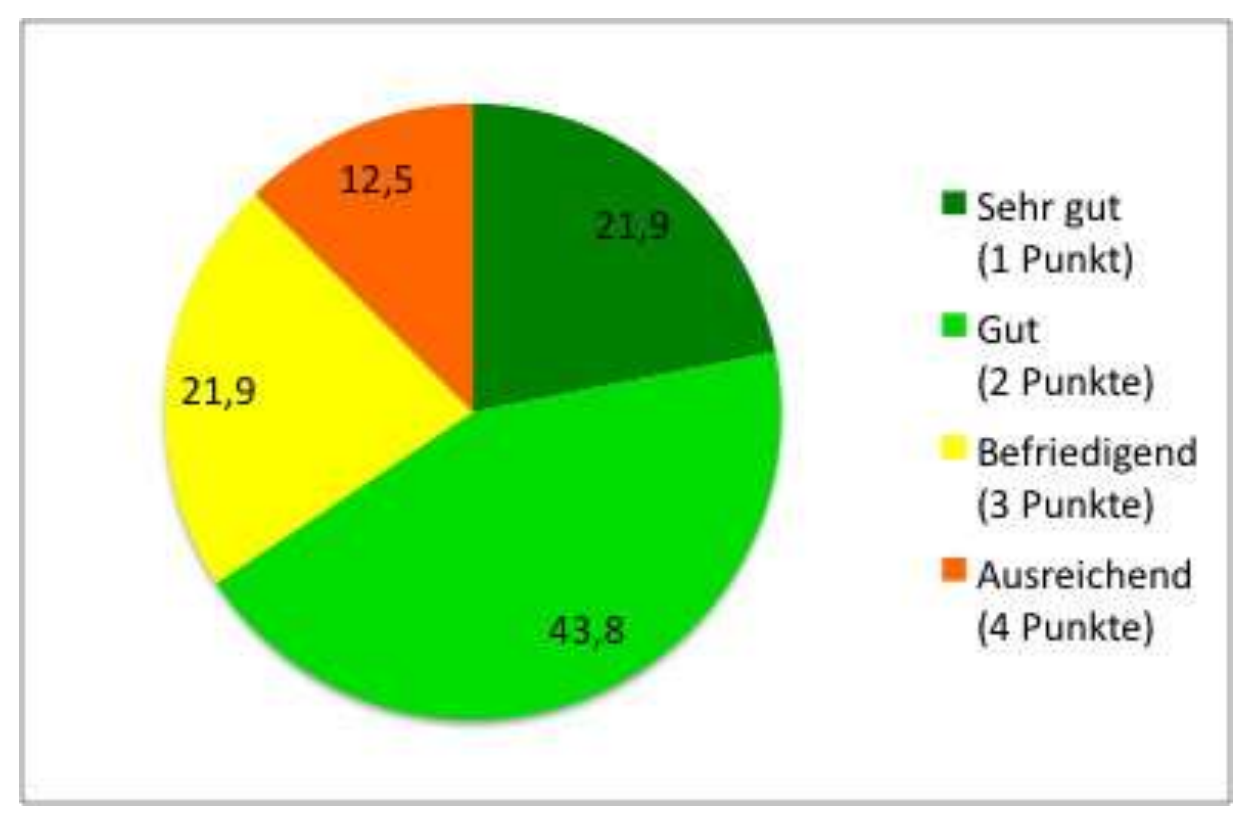

Abbildung 18: Subjektive Beurteilung des Operationsergebnisses durch die Patienten ( $\mathrm{n}=32$ Schultergelenke, Angabe in \%) 
Nur bei 3 Patienten $(9,4 \%)$ fielen die Schmerzen nach ihrer Operation größer aus als erwartet, aber bei keinem Patienten sehr viel stärker als erwartet. Die Schmerzstärke fiel bei 9 Patienten $(28,1 \%)$ genau so wie erwartet aus. D.h., dass die Schmerzen bei insgesamt 20 Patienten (62,5\%) geringer als erwartet waren (Abbildung 19).

Zusammenfassend wurden im Mittel 2,2 \pm 0,9 Punkte vergeben.

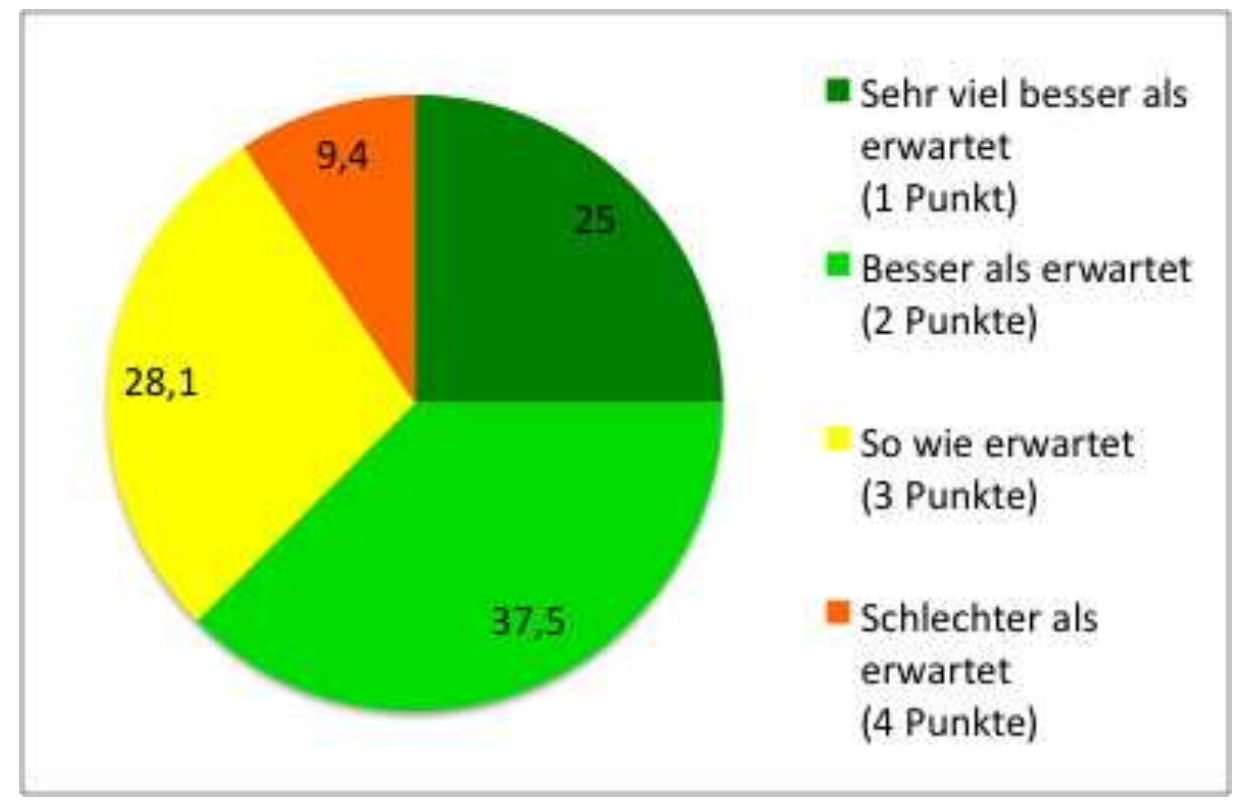

Abbildung 19: Erwartungshaltung Schmerzen $(n=32$ Schultergelenke, Angabe in \%)

Auch die Erwartungen hinsichtlich der Funktion wurden bei der Mehrheit der Patienten erfüllt. 20 Patienten $(62,5 \%)$ beurteilten die Funktion des operierten Schultergelenks sehr viel besser oder besser als erwartet (Abbildung 20). Bei zehn Patienten (31,3\%) fiel sie wie erwartet aus und nur bei zwei Patienten (6,3\%) schlechter als erwartet. Auch hier wurde die vorgegebene Kategorie „sehr viel schlechter als erwartet" in keinem Fall gewählt.

Insgesamt vergaben die Patienten 2,3 \pm 0,8 Punkte. 


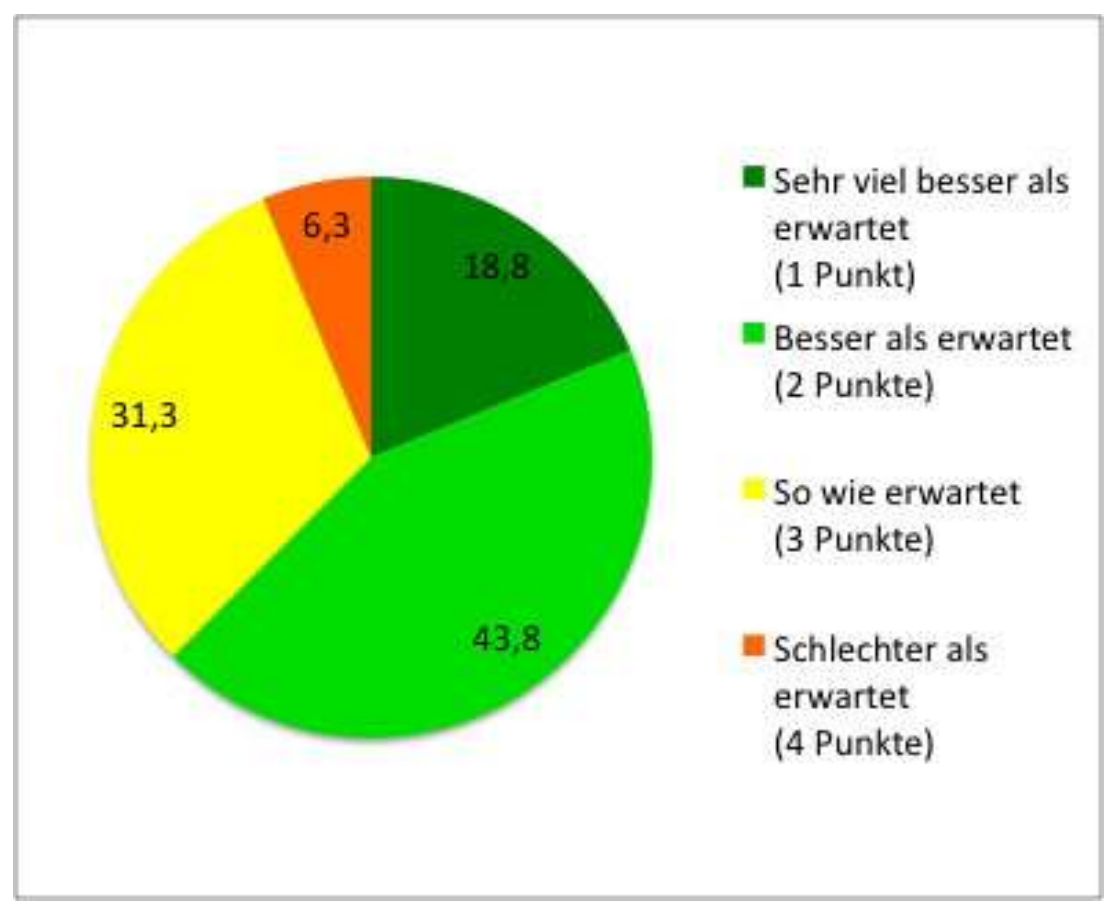

Abbildung 20: Erwartungshaltung Funktion ( $n=32$ Schultergelenke, Angabe in \%)

\subsection{Einfluss des Geschlechts auf die Operationsergebnisse}

\subsubsection{Allgemeine und Operationsdaten}

Die weiblichen Patienten waren mit 73,4 \pm 6,1 Jahren statistisch signifikant älter als die männlichen Patienten mit 66,6 \pm 6,3 Jahren. Bei der Einteilung des Alters in Kategorien waren unter den Frauen 78,9\% älter als 68 Jahre, unter den Männern aber nur 38,5\% $(p<0,05)$.

Beide Geschlechter unterschieden sich nicht in Bezug auf die Körperseite des operierten Schultergelenks, auf die Häufigkeit von Voroperationen oder die Operationsindikation (Tabelle 6). 
Tabelle 6: $\quad$ Allgemeine und Operationsdaten im Vergleich beider Geschlechter

\begin{tabular}{|c|c|c|c|}
\hline & männlich $(n=13)$ & weiblich (n = 19) & p \\
\hline Alter (Jahre) & $66,6 \pm 6,3$ & $73,4 \pm 6,1$ & $<0,05$ \\
\hline \multirow{3}{*}{$\begin{array}{r}\text { Alter in Kategorien } \\
\text { bis } 68 \text { Jahre } \\
\text { über } 68 \text { Jahre }\end{array}$} & & & \\
\hline & $8(61,5 \%)$ & $4(21,1 \%)$ & $<0,05$ \\
\hline & $5(38,5 \%)$ & $15(78,9 \%)$ & \\
\hline \multicolumn{4}{|l|}{ Schultergelenk (Anzahl) } \\
\hline links & $7(53,8 \%)$ & $8(42,1 \%)$ & $>0,05$ \\
\hline rechts & $6(46,2 \%)$ & $11(57,9 \%)$ & \\
\hline \multicolumn{4}{|l|}{ Voroperation (Anzahl) } \\
\hline nein & $12(92,3 \%)$ & $15(79,0 \%)$ & $>0,05$ \\
\hline ja & $1(7,7 \%)$ & $4(21,0 \%)$ & \\
\hline \multicolumn{4}{|l|}{ Indikation (Anzahl) } \\
\hline Omarthrose & $13(100,0 \%)$ & $17(89,4 \%)$ & $\cap 05$ \\
\hline posttraumatisch & 0 & $1(5,3 \%)$ & 0,0 \\
\hline Kopfnekrose & 0 & $1(5,3 \%)$ & \\
\hline
\end{tabular}

\subsubsection{Beweglichkeit}

Weder bezüglich der Abduktion noch der Flexion oder Außenrotation konnten statistisch signifikante Unterschiede zwischen den Ergebnissen bei Männern oder Frauen nachgewiesen werden (Tabelle 7). 
Tabelle 7: Verbesserungen der Beweglichkeit nach dem Oberflächenersatz des Schultergelenks in Abhängkeit vom Geschlecht der Patienten

\begin{tabular}{|l|c|c|c|}
\hline Abduktion $\left(^{\circ}\right)$ & männlich $(\mathbf{n}=\mathbf{1 3})$ & weiblich $(\mathbf{n}=\mathbf{1 9})$ & $\mathbf{p}$ \\
\hline präoperativ & $68,5 \pm 28,5$ & $69,5 \pm 31,2$ & $>0,05$ \\
\hline postoperativ & $123,1 \pm 23,6$ & $115,8 \pm 19,8$ & $>0,05$ \\
\hline Differenz & $54,6 \pm 25,3$ & $46,3 \pm 24,2$ & $>0,05$ \\
\hline Flexion ( $\left.{ }^{\circ}\right)$ & männlich $(\mathbf{n}=\mathbf{1 3})$ & weiblich $(\mathbf{n}=\mathbf{1 9})$ & $\mathbf{p}$ \\
\hline präoperativ & $81,5 \pm 31,8$ & $81,6 \pm 31,5$ & $>0,05$ \\
\hline postoperativ & $124,6 \pm 24,0$ & $122,1 \pm 24,9$ & $>0,05$ \\
\hline Differenz & $43,1 \pm 28,9$ & $40,5 \pm 28,6$ & $>0,05$ \\
\hline Außenrotation $\left(^{\circ}\right)$ & männlich $(\mathbf{n}=\mathbf{1 3})$ & weiblich $(\mathbf{n}=\mathbf{1 9})$ & $\mathbf{p}$ \\
\hline präoperativ & $20,8 \pm 16,6$ & $24,5 \pm 16,1$ & $>0,05$ \\
\hline postoperativ & $43,8 \pm 13,9$ & $41,8 \pm 13,8$ & $>0,05$ \\
\hline Differenz & $23,0 \pm 31,1$ & $17,3 \pm 14,7$ & $>0,05$ \\
\hline
\end{tabular}

\subsubsection{Constant-Score}

Auch hinsichtlich des Constant-Scores ergaben sich weder für das Gesamtergebnis noch für die einzelnen Kategorien statistisch signifikante Unterschiede zwischen den Ergebnissen von Männern und Frauen (Tabelle 8). 
Tabelle 8: Veränderungen der Einzelparameter des Constant-Scores sowie des Gesamtscores in Abhängigkeit vom Geschlecht (Angabe in Punkten; MW \pm SD)

\begin{tabular}{|c|c|c|c|}
\hline Schmerzen & männlich (n = 13) & weiblich ( $n=19)$ & $\mathbf{p}$ \\
\hline präoperativ & $7,3 \pm 2,2$ & $7,2 \pm 2,1$ & $>0,05$ \\
\hline postoperativ & $10,5 \pm 2,2$ & $9,9 \pm 2,4$ & $>0,05$ \\
\hline Differenz & $3,2 \pm 1,6$ & $2,8 \pm 2,5$ & $>0,05$ \\
\hline Aktivität & männlich (n = 13) & weiblich $(n=19)$ & $\mathbf{p}$ \\
\hline präoperativ & $9,1 \pm 2,8$ & $8,5 \pm 2,7$ & $>0,05$ \\
\hline postoperativ & $12,5 \pm 2,2$ & $12,1 \pm 3,3$ & $>0,05$ \\
\hline Differenz & $3,5 \pm 1,9$ & $3,5 \pm 3,7$ & $>0,05$ \\
\hline Beweglichkeit & männlich (n = 13) & weiblich $(n=19)$ & p \\
\hline präoperativ & $15,0 \pm 5,8$ & $16,1 \pm 6,1$ & $>0,05$ \\
\hline postoperativ & $24,5 \pm 6,6$ & $23,6 \pm 6,4$ & $>0,05$ \\
\hline Differenz & $9,5 \pm 6,6$ & $7,5 \pm 4,5$ & $>0,05$ \\
\hline Kraft & männlich (n = 13) & weiblich ( $n=19$ ) & $\mathbf{p}$ \\
\hline präoperativ & $13,5 \pm 4,2$ & $12,5 \pm 2,0$ & $>0,05$ \\
\hline postoperativ & $16,2 \pm 4,3$ & $14,2 \pm 4,0$ & $>0,05$ \\
\hline Differenz & $2,5 \pm 3,4$ & $1,7 \pm 2,8$ & $>0,05$ \\
\hline Gesamtscore & männlich (n = 13) & weiblich ( $n=19)$ & $\mathbf{p}$ \\
\hline präoperativ & $45,0 \pm 12,8$ & $44,3 \pm 9,2$ & $>0,05$ \\
\hline postoperativ & $63,8 \pm 13,1$ & $59,8 \pm 13,2$ & $>0,05$ \\
\hline Differenz & $18,8 \pm 10,3$ & $15,5 \pm 10,6$ & $>0,05$ \\
\hline
\end{tabular}




\subsubsection{DASH-Score}

Männer und Frauen beurteilten das Operationsergebnis nach dem DASH-Score nicht statistisch signifikant unterschiedlich (Tabelle 9, Tabelle 10). Auch die Erwartungshaltungen zu Schmerzen und Funktion fielen nicht abweichend voneinander aus.

Auffallend ist, dass bei $84,6 \%$ der Männer, aber nur bei 47,4\% der Frauen die Funktion der operierten Schulter besser als erwartet ausfiel. Der Unterschied verfehlte mit $p=0,08$ knapp die Schwelle zur statistischen Signifikanz.

Tabelle 9: $\quad$ Mittlere Punktzahlen im DASH-Score bei Männern und Frauen

\begin{tabular}{|l|c|c|c|}
\hline & $\begin{array}{c}\text { männlich } \\
(\mathrm{n}=13)\end{array}$ & $\begin{array}{c}\text { weiblich } \\
(\mathrm{n}=19)\end{array}$ & $\mathrm{p}$ \\
\hline Subjektive Beurteilung & $2,2 \pm 1,0$ & $2,3 \pm 0,9$ & $>0,05$ \\
\hline Erwartungshaltung Schmerzen & $2,2 \pm 0,8$ & $2,3 \pm 1,0$ & $>0,05$ \\
\hline Erwartungshaltung Funktion & $2,0 \pm 0,6$ & $2,4 \pm 1,0$ & $>0,05$ \\
\hline
\end{tabular}

Tabelle 10: Beurteilung der Operationsergebnisse nach dem DASH-Score durch Männer und Frauen

\begin{tabular}{|c|c|c|c|}
\hline Subjektive Beurteilung & $\begin{array}{c}\text { männlich } \\
(\mathrm{n}=13)\end{array}$ & $\begin{array}{c}\text { weiblich } \\
(n=19)\end{array}$ & $\mathbf{p}$ \\
\hline $\begin{array}{l}\text { Sehr gut } \\
\text { (1 Punkt) }\end{array}$ & $\begin{array}{c}3 \\
(23,1 \%) \\
\end{array}$ & $\begin{array}{c}4 \\
(21,2 \%) \\
\end{array}$ & \multirow{5}{*}{$>0,05$} \\
\hline $\begin{array}{l}\text { Gut } \\
\text { (2 Punkte) }\end{array}$ & $\begin{array}{c}6 \\
(46,2 \%) \\
\end{array}$ & $\begin{array}{c}8 \\
(42,1 \%) \\
\end{array}$ & \\
\hline $\begin{array}{l}\text { Befriedigend } \\
\text { (3 Punkte) }\end{array}$ & $\begin{array}{c}2 \\
(15,4 \%)\end{array}$ & $\begin{array}{c}5 \\
(26,3 \%) \\
\end{array}$ & \\
\hline $\begin{array}{l}\text { Ausreichend } \\
\text { (4 Punkte) }\end{array}$ & $\begin{array}{c}2 \\
(15,4 \%) \\
\end{array}$ & $\begin{array}{c}2 \\
(10,5 \%) \\
\end{array}$ & \\
\hline $\begin{array}{l}\text { Mangelhaft } \\
\text { (5 Punkte) }\end{array}$ & 0 & 0 & \\
\hline
\end{tabular}




\begin{tabular}{|c|c|c|c|}
\hline Erwartungshaltung Schmerzen & $\begin{array}{l}\text { männlich } \\
(\mathrm{n}=13)\end{array}$ & $\begin{array}{l}\text { weiblich } \\
(n=19)\end{array}$ & $\mathbf{p}$ \\
\hline $\begin{array}{l}\text { Sehr viel besser als erwartet } \\
\text { (1 Punkt) }\end{array}$ & $\begin{array}{c}2 \\
(15,4 \%) \\
\end{array}$ & $\begin{array}{c}6 \\
(31,6 \%) \\
\end{array}$ & \multirow{5}{*}{$>0,05$} \\
\hline $\begin{array}{l}\text { Besser als erwartet } \\
\text { ( } 2 \text { Punkte) }\end{array}$ & $\begin{array}{c}8 \\
(61,5 \%) \\
\end{array}$ & $\begin{array}{c}4 \\
(21,1 \%) \\
\end{array}$ & \\
\hline $\begin{array}{l}\text { So wie erwartet } \\
\text { (3 Punkte) }\end{array}$ & $\begin{array}{c}2 \\
(15,4 \%) \\
\end{array}$ & $\begin{array}{c}7 \\
(36,8 \%) \\
\end{array}$ & \\
\hline $\begin{array}{l}\text { Schlechter als erwartet } \\
\text { (4 Punkte) }\end{array}$ & $\begin{array}{c}1 \\
(7,7 \%)\end{array}$ & $\begin{array}{c}2 \\
(10,5 \%) \\
\end{array}$ & \\
\hline $\begin{array}{l}\text { Sehr viel schlechter als erwartet } \\
\text { (5 Punkte) }\end{array}$ & 0 & 0 & \\
\hline Erwartungshaltung Funktion & $\begin{array}{c}\text { männlich } \\
(\mathrm{n}=13)\end{array}$ & $\begin{array}{l}\text { weiblich } \\
(n=19)\end{array}$ & $\mathbf{p}$ \\
\hline $\begin{array}{l}\text { Sehr viel besser als erwartet } \\
\text { (1 Punkt) }\end{array}$ & $\begin{array}{c}2 \\
(15,4 \%) \\
\end{array}$ & $\begin{array}{c}4 \\
(21,1 \%) \\
\end{array}$ & \multirow{5}{*}{$\begin{array}{l}>0,05 \\
(0,08)\end{array}$} \\
\hline $\begin{array}{l}\text { Besser als erwartet } \\
\text { ( } 2 \text { Punkte) }\end{array}$ & $\begin{array}{c}9 \\
(69,2 \%) \\
\end{array}$ & $\begin{array}{c}5 \\
(26,3 \%)\end{array}$ & \\
\hline $\begin{array}{l}\text { So wie erwartet } \\
\text { (3 Punkte) }\end{array}$ & $\begin{array}{c}2 \\
(15,4 \%) \\
\end{array}$ & $\begin{array}{c}8 \\
(42,1 \%) \\
\end{array}$ & \\
\hline $\begin{array}{l}\text { Schlechter als erwartet } \\
\text { (4 Punkte) }\end{array}$ & 0 & $\begin{array}{c}2 \\
(10,5 \%) \\
\end{array}$ & \\
\hline $\begin{array}{l}\text { Sehr viel schlechter als erwartet } \\
\text { ( } 5 \text { Punkte) }\end{array}$ & 0 & 0 & \\
\hline
\end{tabular}

\subsection{Einfluss des Alters auf die Operationsergebnisse}

\subsubsection{Operationsdaten}

Das rechte Schultergelenk war bei $75,0 \%$ der jüngeren Patienten $(n=9)$, aber nur bei $40,0 \%$ der älteren Patienten $(n=8)$ operiert worden. Der Unterschied zwischen beiden Altersgruppen war nicht statistisch signifikant (Tabelle 11). 
Hinsichtlich des Vorkommens von Voroperationen und der Operationsindikationen unterschieden sich beide Altersgruppen nicht.

Tabelle 11: Operationsdaten im Vergleich beider Geschlechter

\begin{tabular}{|c|c|c|c|}
\hline & $\begin{array}{c}\text { Alter bis } 68 \text { Jahre } \\
\qquad(n=12)\end{array}$ & $\begin{array}{c}\text { Alter über } 68 \text { Jahre } \\
\qquad(\mathrm{n}=20)\end{array}$ & $\mathbf{p}$ \\
\hline \multirow{3}{*}{$\begin{array}{r}\text { Schultergelenk (Anzahl) } \\
\text { links } \\
\text { rechts }\end{array}$} & & & \\
\hline & $3(25,0 \%)$ & $12(60,0 \%)$ & $>0,05$ \\
\hline & $9(75,0 \%)$ & $8(40,0 \%)$ & \\
\hline \multirow{3}{*}{$\begin{array}{r}\text { Voroperation (Anzahl) } \\
\text { nein } \\
\text { ja }\end{array}$} & & & \\
\hline & $10(83,3 \%)$ & $17(85,0 \%)$ & $>0,05$ \\
\hline & $2(16,7 \%)$ & $3(15,0 \%)$ & \\
\hline \multirow{3}{*}{$\begin{array}{r}\text { Indikation (Anzahl) } \\
\text { Omarthrose } \\
\text { posttraumatisch }\end{array}$} & & & \\
\hline & $12(100,0 \%)$ & $18(90,0 \%)$ & $\cap 05$ \\
\hline & 0 & $1(5,0 \%)$ & \\
\hline Kopfnekrose & 0 & $1(5,0 \%)$ & \\
\hline
\end{tabular}

\subsubsection{Beweglichkeit}

Weder bezüglich der Abduktion noch der Flexion oder Außenrotation konnten statistisch signifikante altersabhängige Unterschiede nachgewiesen werden (Tabelle 7). 
Tabelle 12: Verbesserungen der Beweglichkeit nach dem Oberflächenersatz des Schultergelenks in Abhängkeit vom Alter der Patienten

\begin{tabular}{|c|c|c|c|}
\hline Abduktion $\left({ }^{\circ}\right)$ & $\begin{array}{c}\text { Alter bis } 68 \text { Jahre } \\
\qquad(\mathrm{n}=12)\end{array}$ & $\begin{array}{c}\text { Alter über } 68 \text { Jahre } \\
\qquad(\mathrm{n}=20)\end{array}$ & p \\
\hline präoperativ & $81,7 \pm 29,2$ & $61,5 \pm 28,0$ & $>0,05$ \\
\hline postoperativ & $120,0 \pm 30,5$ & $118,0 \pm 14,4$ & $>0,05$ \\
\hline Differenz & $38,3 \pm 31,2$ & $56,5 \pm 27,0$ & $>0,05$ \\
\hline Flexion $\left({ }^{\circ}\right)$ & $\begin{array}{l}\text { Alter bis } 68 \text { Jahre } \\
\qquad(n=12)\end{array}$ & $\begin{array}{l}\text { Alter über } 68 \text { Jahre } \\
\qquad(\mathrm{n}=20)\end{array}$ & $\mathbf{p}$ \\
\hline präoperativ & $87,1 \pm 37,1$ & $78,3 \pm 27,4$ & $>0,05$ \\
\hline postoperativ & $124,2 \pm 29,4$ & $122,5 \pm 21,2$ & $>0,05$ \\
\hline Differenz & $37,1 \pm 33,2$ & $44,2 \pm 23,6$ & $>0,05$ \\
\hline Außenrotation $\left({ }^{\circ}\right)$ & $\begin{array}{c}\text { Alter bis } 68 \text { Jahre } \\
\qquad(\mathrm{n}=12)\end{array}$ & $\begin{array}{c}\text { Alter über } 68 \text { Jahre } \\
(\mathrm{n}=20)\end{array}$ & $\mathbf{p}$ \\
\hline präoperativ & $26,3 \pm 14,3$ & $21,0 \pm 17,2$ & $>0,05$ \\
\hline postoperativ & $42,1 \pm 15,9$ & $43,0 \pm 12,5$ & $>0,05$ \\
\hline Differenz & $15,8 \pm 15,7$ & $22,0 \pm 15,8$ & $>0,05$ \\
\hline
\end{tabular}

\subsubsection{Constant-Score}

Bei der Beurteilung der Schmerzen nach dem Constant-Score war die Differenz zwischen prä- und postoperativen Schmerzen statistisch signifikant unterschiedlich: Patienten im Alter über 68 Jahren erfuhren eine größere Schmerzlinderung als jüngere Patienten (Tabelle 8).

Bei den übrigen Kategorien des Constant-Scores und dem Gesamtscore ergaben sich keine altersabhängigen Unterschiede. 
Tabelle 13: Veränderungen der Einzelparameter des Constant-Scores sowie des Gesamtscores in Abhängigkeit vom Alter (Angabe in Punkten; MW \pm SD)

\begin{tabular}{|c|c|c|c|}
\hline Schmerzen & $\begin{array}{l}\text { Alter bis } 68 \text { Jahre } \\
\qquad(n=12)\end{array}$ & $\begin{array}{l}\text { Alter über } 68 \text { Jahre } \\
\qquad(\mathrm{n}=20)\end{array}$ & p \\
\hline präoperativ & $8,1 \pm 2,3$ & $6,8 \pm 1,9$ & $>0,05$ \\
\hline postoperativ & $9,9 \pm 3,1$ & $10,4 \pm 1,6$ & $>0,05$ \\
\hline Differenz & $1,8 \pm 2,2$ & $3,6 \pm 1,8$ & $<0,05$ \\
\hline Aktivität & $\begin{array}{c}\text { Alter bis } 68 \text { Jahre } \\
\qquad(n=12)\end{array}$ & $\begin{array}{l}\text { Alter über } 68 \text { Jahre } \\
\qquad(n=20)\end{array}$ & p \\
\hline präoperativ & $9,3 \pm 2,6$ & $8,5 \pm 2,8$ & $>0,05$ \\
\hline postoperativ & $11,8 \pm 3,0$ & $12,6 \pm 2,8$ & $>0,05$ \\
\hline Differenz & $2,5 \pm 2,2$ & $4,1 \pm 3,4$ & $>0,05$ \\
\hline Beweglichkeit & $\begin{array}{l}\text { Alter bis } 68 \text { Jahre } \\
\qquad(n=12)\end{array}$ & $\begin{array}{l}\text { Alter über } 68 \text { Jahre } \\
\qquad(n=20)\end{array}$ & p \\
\hline präoperativ & $16,4 \pm 4,2$ & $15,2 \pm 6,8$ & $>0,05$ \\
\hline postoperativ & $23,5 \pm 7,8$ & $24,3 \pm 5,6$ & $>0,05$ \\
\hline Differenz & $7,1 \pm 7,2$ & $2,1 \pm 2,9$ & $>0,05$ \\
\hline Kraft & $\begin{array}{l}\text { Alter bis } 68 \text { Jahre } \\
\qquad(n=12)\end{array}$ & $\begin{array}{l}\text { Alter über } 68 \text { Jahre } \\
\qquad(\mathrm{n}=20)\end{array}$ & p \\
\hline präoperativ & $13,8 \pm 2,8$ & $12,4 \pm 3,2$ & $>0,05$ \\
\hline postoperativ & $15,9 \pm 4,6$ & $14,5 \pm 3,9$ & $>0,05$ \\
\hline Differenz & $2,1 \pm 3,4$ & $2,1 \pm 2,9$ & $>0,05$ \\
\hline Gesamtscore & $\begin{array}{l}\text { Alter bis } 68 \text { Jahre } \\
\qquad(n=12)\end{array}$ & $\begin{array}{l}\text { Alter über } 68 \text { Jahre } \\
\qquad(n=20)\end{array}$ & p \\
\hline präoperativ & $47,6 \pm 8,9$ & $42,8 \pm 11,4$ & $>0,05$ \\
\hline postoperativ & $61,1 \pm 16,8$ & $61,6 \pm 10,8$ & $>0,05$ \\
\hline Differenz & $13,5 \pm 12,4$ & $18,8 \pm 8,9$ & $>0,05$ \\
\hline
\end{tabular}




\subsubsection{DASH-Score}

Das Operationsergebnis nach dem DASH-Score wurde durch die älteren oder jüngeren Patienten nicht statistisch signifikant unterschiedlich beurteilt (Tabelle 9, Tabelle 10). Auch die Erwartungshaltungen zu Schmerzen und Funktion fielen bei beiden Altersgruppen nicht abweichend voneinander aus (Tabelle 15).

Tabelle 14: Mittlere Punktzahlen im DASH-Score in Abhängigkeit vom Alter

\begin{tabular}{|l|c|c|c|}
\hline & $\begin{array}{c}\text { Alter bis } \mathbf{6 8} \mathbf{~ J .} \\
(\mathbf{n = 1 2})\end{array}$ & $\begin{array}{c}\text { Alter über 68 J. } \\
(\mathbf{n = 2 0})\end{array}$ & $\mathrm{p}$ \\
\hline Subjektive Beurteilung & $2,3 \pm 1,0$ & $2,2 \pm 1,0$ & $>0,05$ \\
\hline Erwartungshaltung Schmerzen & $2,5 \pm 0,9$ & $2,1 \pm 0,9$ & $>0,05$ \\
\hline Erwartungshaltung Funktion & $2,4 \pm 0,7$ & $2,2 \pm 0,9$ & $>0,05$ \\
\hline
\end{tabular}

Tabelle 15: Beurteilung der Operationsergebnisse nach dem DASH-Score in Abhängigkeit vom Alter

\begin{tabular}{|c|c|c|c|}
\hline Subjektive Beurteilung & $\begin{array}{c}\text { Alter bis } 68 \mathrm{~J} . \\
\quad(\mathrm{n}=12)\end{array}$ & $\begin{array}{l}\text { Alter über } 68 \mathrm{~J} . \\
\qquad(\mathrm{n}=20)\end{array}$ & $\mathbf{p}$ \\
\hline $\begin{array}{l}\text { Sehr gut } \\
\text { (1 Punkt) }\end{array}$ & $\begin{array}{c}2 \\
(16,7 \%) \\
\end{array}$ & $\begin{array}{c}5 \\
(25,0 \%) \\
\end{array}$ & \multirow{5}{*}{$>0,05$} \\
\hline $\begin{array}{l}\text { Gut } \\
\text { (2 Punkte) }\end{array}$ & $\begin{array}{c}6 \\
(50,0 \%)\end{array}$ & $\begin{array}{c}8 \\
(40,0 \%)\end{array}$ & \\
\hline $\begin{array}{l}\text { Befriedigend } \\
\text { (3 Punkte) }\end{array}$ & $\begin{array}{c}2 \\
(16,7 \%) \\
\end{array}$ & $\begin{array}{c}5 \\
(25,0 \%) \\
\end{array}$ & \\
\hline $\begin{array}{l}\text { Ausreichend } \\
\text { (4 Punkte) }\end{array}$ & $\begin{array}{c}2 \\
(16,7 \%) \\
\end{array}$ & $\begin{array}{c}2 \\
(10,0 \%) \\
\end{array}$ & \\
\hline $\begin{array}{l}\text { Mangelhaft } \\
\text { (5 Punkte) }\end{array}$ & 0 & 0 & \\
\hline
\end{tabular}




\begin{tabular}{|c|c|c|c|}
\hline Erwartungshaltung Schmerzen & $\begin{array}{c}\text { Alter bis } 68 \mathrm{~J} . \\
(\mathrm{n}=12)\end{array}$ & $\begin{array}{l}\text { Alter über } 68 \mathrm{~J} . \\
(\mathrm{n}=20)\end{array}$ & $\mathbf{p}$ \\
\hline $\begin{array}{l}\text { Sehr viel besser als erwartet } \\
\text { (1 Punkt) }\end{array}$ & $\begin{array}{c}1 \\
(8,3 \%) \\
\end{array}$ & $\begin{array}{c}7 \\
(35,0 \%) \\
\end{array}$ & \multirow{5}{*}{$>0,05$} \\
\hline $\begin{array}{l}\text { Besser als erwartet } \\
\text { ( } 2 \text { Punkte) }\end{array}$ & $\begin{array}{c}6 \\
(50,0 \%) \\
\end{array}$ & $\begin{array}{c}6 \\
(30,0 \%) \\
\end{array}$ & \\
\hline $\begin{array}{l}\text { So wie erwartet } \\
\text { (3 Punkte) }\end{array}$ & $\begin{array}{c}3 \\
(25,0 \%)\end{array}$ & $\begin{array}{c}6 \\
(30,0 \%)\end{array}$ & \\
\hline $\begin{array}{l}\text { Schlechter als erwartet } \\
\text { (4 Punkte) }\end{array}$ & $\begin{array}{c}2 \\
(16,7 \%) \\
\end{array}$ & $\begin{array}{c}1 \\
(5,0 \%) \\
\end{array}$ & \\
\hline $\begin{array}{l}\text { Sehr viel schlechter als erwartet } \\
\text { (5 Punkte) }\end{array}$ & 0 & 0 & \\
\hline Erwartungshaltung Funktion & $\begin{array}{l}\text { Alter bis } 68 \mathrm{~J} . \\
\quad(n=12)\end{array}$ & $\begin{array}{l}\text { Alter über } 68 \mathrm{~J} . \\
\qquad(\mathrm{n}=20)\end{array}$ & $\mathbf{p}$ \\
\hline $\begin{array}{l}\text { Sehr viel besser als erwartet } \\
\text { (1 Punkt) }\end{array}$ & 0 & $\begin{array}{c}6 \\
(30,0 \%) \\
\end{array}$ & \multirow{5}{*}{$>0,05$} \\
\hline $\begin{array}{l}\text { Besser als erwartet } \\
\text { ( } 2 \text { Punkte) }\end{array}$ & $\begin{array}{c}8 \\
(66,7 \%)\end{array}$ & $\begin{array}{c}6 \\
(30,0 \%)\end{array}$ & \\
\hline $\begin{array}{l}\text { So wie erwartet } \\
\text { (3 Punkte) }\end{array}$ & $\begin{array}{c}3 \\
(25,0 \%) \\
\end{array}$ & $\begin{array}{c}7 \\
(35,0 \%) \\
\end{array}$ & \\
\hline $\begin{array}{l}\text { Schlechter als erwartet } \\
\text { (4 Punkte) }\end{array}$ & $\begin{array}{c}1 \\
(8,3 \%) \\
\end{array}$ & $\begin{array}{c}1 \\
(5,0 \%) \\
\end{array}$ & \\
\hline $\begin{array}{l}\text { Sehr viel schlechter als erwartet } \\
\text { ( } 5 \text { Punkte) }\end{array}$ & 0 & 0 & \\
\hline
\end{tabular}

\subsection{Radiologische Nachuntersuchung}

Zur radiologischen Nachuntersuchung standen 26 Patienten (29 Schultergelenke) zur Verfügung. Bei 20 Patienten beziehungsweise 23 Schultergelenken befanden sich die Durom-Cup-Prothesen bei der Nachuntersuchung in regelrechter Lage und wiesen keinerlei Anzeichen einer Lockerung, degenerativer Veränderungen oder einer Luxation auf. 
Bei sechs Patienten/Schultergelenken waren folgende Abweichungen vom radiologischen Normalbefund erkennbar:

○ zwei Patienten/Schultergelenke: ausgeprägte Sklerosierung im Glenoid-Bereich

○ zwei Patienten/Schultergelenke: Humeruskopf-Hochstand

○ ein Patient/Schultergelenk: periartikuläre Ossifikation

○ ein Patient/Schultergelenk: Lockerungszeichen, Lysesaum-Bildung und Humeruskopf-Hochstand 


\section{Diskussion}

Der Oberflächenersatz des Humerus hat sich bei oberflächlichen Degenerationen unterschiedlicher Genese klinisch bewährt. In vergleichenden Studien wurden Ergebnisse erzielt, die denjenigen von Totalendoprothesen gleichwertig sind (Levy und Copeland, 2004; Levy et al., 2004; Mullett et al., 2007; Buchner et al., 2008; Scalise et al., 2008). Die Studienlage zu den klinischen Erfahrungen mit dem Oberflächenersatz ist jedoch leider unbefriedigend. Von den zahlreichen Prothesenvarianten ist nur der CopelandCup, der zementfrei implantiert wird, vergleichsweise gut untersucht (Levy und Copeland, 2001, 2004; Levy et al., 2004; Thomas et al., 2005; Mullett et al., 2007; Buchner et al., 2008; Jerosch et al., 2008; Scalise et al., 2008; Raiss et al., 2009; Raiss et al., 2010). Über zementierbare Prothesen wurde bis zum Jahr 2000 lediglich über Erfahrungen mit der Scan-Shoulder berichtet (Jonsson et al., 1988; Rydholm und Sjögren, 1993; Alund et al., 2000), von denen eine Studie lediglich fünf Patienten mit einem Oberflächenersatz weiteren fünf Patienten mit einer Arthrodese gegenüberstellt.

Bei der Scan-Shoulder handelt es sich um eine Edelstahlkappe, die außer ihrem Oberflächenrelief auf der Innenseite keine weiteren Verankerungselemente aufweist. Bei der Untersuchung von 39 Patienten mit rheumatoider Arthritis wurden nach durchschnittlich 4,4 Jahren eine mittlere proximale Humerusmigration um 5,2 mm, eine mittlere Abnahme der Inklination um 5,2 $2^{\circ}$ und bei zahlreichen Schultergelenken eine Verringerung des Abstandes über der Tuberositas um $3 \mathrm{~mm}$ und mehr festgestellt. Gleichzeitig bestand eine Zunahme glenoidaler Erosionen. Obwohl diese Veränderungen nicht mit der Schulterfunktion oder Schmerzen korreliert waren, stellten die Autoren die Eignung des Scan-Cups für Patienten mit einer rheumatoiden Arthritis in Frage (Alund et al., 2000).

Mit dem Durom-Cup wurde ein zementierbarer Oberflächenersatz entwickelt, der durch seine Oberflächengestaltung und einen zentralen Verankerungszapfen (Rüther, 2002) eine größere Stabilität gewährleisten soll. Zum Durom-Cup liegen bisher vier klinische Studien vor (Fink et al., 2004; Fürst et al., 2007, 2008; Fakler et al., 2010) - davon zwei mit identischem Patientengut (Fürst et al., 2007, 2008) -, die sich überwiegend mit den Behandlungsergebnissen von Patienten mit einer rheumatoiden Arthritis beschäftigten. In der vorliegenden Untersuchung wurden die Resultate nach dem humeralen Oberflächenersatz durch einen Durom-Cup bei 29 Patienten (32 Schultergelen- 
ke) mit einer Omarthrose ausgewertet. Die Nachuntersuchungen fanden nach durchschnittlich 2,7 $\pm 2,1$ Jahren $(0,5$ bis 9 Jahre statt).

Bei der Nachuntersuchung hatte sich der Bewegungsumfang der operierten Schultern statistisch signifikant vergrößert und erfüllte die Zielkriterien von Neer et al. (1982) nach einer postoperativ zu erreichenden Abduktion von mindestens $90^{\circ}$ beziehungsweise Außenrotation von mindestens $20^{\circ}$. Das Ausmaß der Abduktion hatte sich um $49,7 \pm 25,8^{\circ}$, der Flexion um 41,5 $\pm 28,2^{\circ}$ und der Außenrotation um 19,7 $\pm 15,3^{\circ}$ gesteigert. Diese Ergebnisse stimmen sehr gut mit denjenigen der Studie von Fakler et al. (2010) überein. Dort waren 52 Schultergelenke (49 Patienten) mit einer Durom-CupProthese versorgt worden. Als Indikationen wurden eine primäre Omarthrose (69\%), eine rheumatoide Arthritis (14\%), eine posttraumatische Omarthrose (6\%) sowie eine Humeruskopfnekrose oder Chondromatose (je 2\%) genannt. In die übrigen Studien zum Durom-Cup von Fink et al., 2004 und Fürst et al., 2007 (Patienten identisch mit Fürst et al., 2008) waren ausschließlich Patienten mit einer rheumatoiden Arthritis eingeschlossen und je nach dem Schweregrad der Grunderkrankung drei Untergruppen gebildet worden: intakte Rotatorenmanschette $(\mathrm{A})$, partieller oder reparierter Defekt der Rotatorenmanschette (B) und massiver Defekt der Rotatorenmanschette (C). Unabhängig vom Zustand der Rotatorenmanschette waren etwa gleich gute Verbesserungen der Beweglichkeit durch das Einsetzen des Oberflächenersatzes erzielt worden (Abbildung 21). Vergleichbare Ergebnisse waren auch nach der Implantation einer nicht zementierten Copeland-Kappe berichtet worden (Levy und Copeland, 2001, 2004; Levy et al., 2004; Thomas et al., 2005; Buchner et al., 2008; Raiss et al., 2009; Raiss et al., 2010). Die Studien zur Scan-Shoulder erlaubten keine direkten Vergleich mit den eigenen Ergebnissen, jedoch wurden auch hier übereinstimmend deutliche Steigerungen des Bewegungsumfanges im Rahmen der jeweiligen Nachuntersuchungen beschrieben (Jonsson et al., 1988; Rydholm und Sjögren, 1993; Alund et al., 2000). 


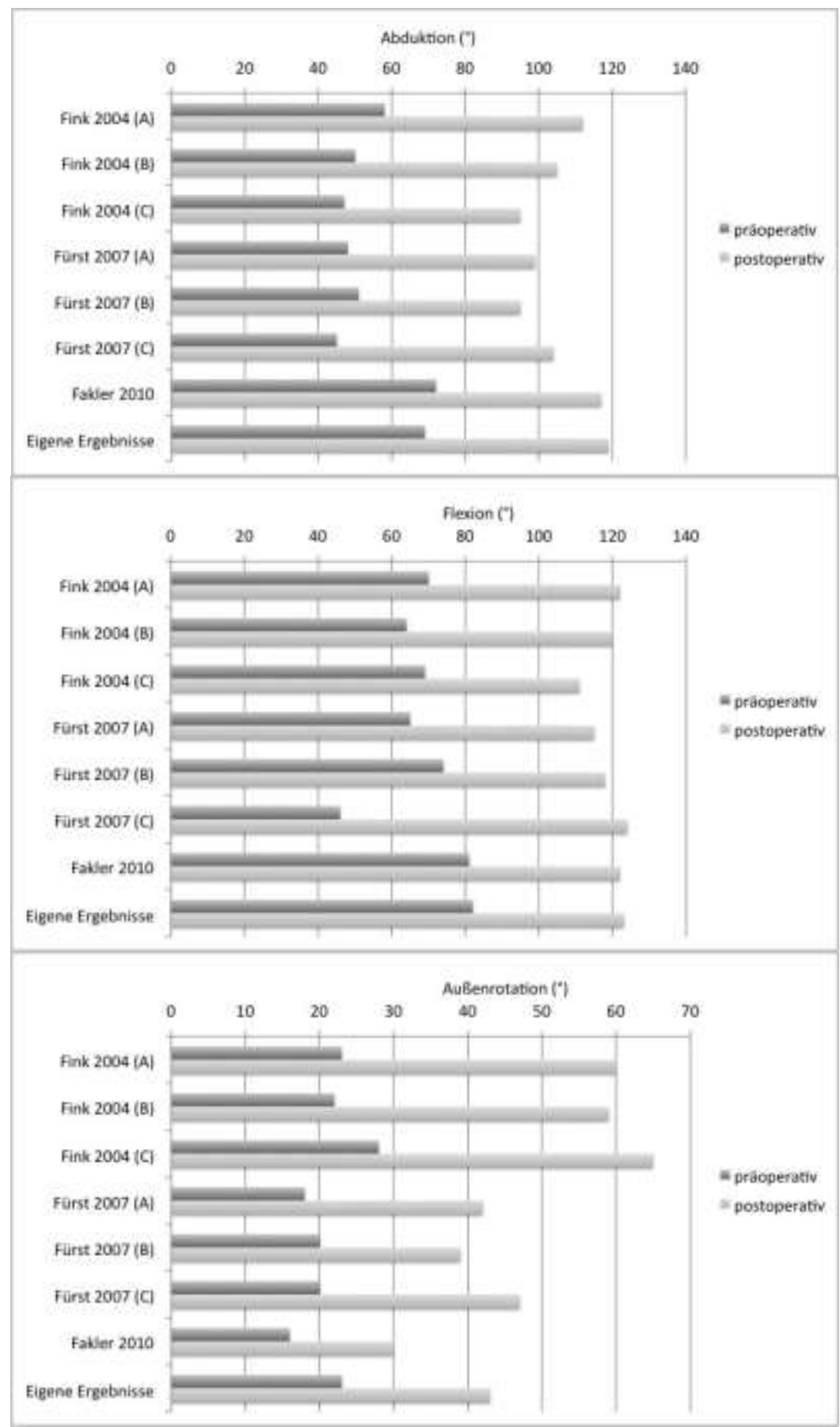

Abbildung 21: Entwicklung des Bewegungsausmaßes der operierten Schultergelenke mit einem Durom-Cup-Oberflächenersatz - Vergleich der eigenen Ergebnisse mit Literaturangaben 
Neben einer mechanischen Erleichterung der Gelenkbeweglichkeit kommt besonders der Schmerzreduzierung Bedeutung hinsichtlich der Verbesserung der Funktionalität zu. Im Rahmen der Beurteilung mittels Constant-Score wurden in der eigenen Untersuchungen die Schmerzen der Patienten in vier Kategorien eingeteilt ( 0 Punkte $=$ schwere, 5 Punkte = mäßige, 10 Punkte $=$ leichte und 15 Punkte $=$ keine Schmerzen). Präoperativ wiesen unsere Patienten im Mittel 7,3 $\pm 2,1$ Punkte auf, bei der Nachuntersuchung hatte sich die Schmerzsymptomatik statistisch hoch signifikant auf 10,2 $\pm 2,2$ Punkte verbessert $(p<0,0001)$. Die Schmerzreduzierung entsprach etwa derjenigen, die Buchner et al. (2008) bei ihren Patienten mit einer Omarthrose nach Implantation eines Copeland-Cups beobachtet hatten. Dort betrug die anfängliche Schmerzstärke 4,1 \pm 2,2 Punkte und die postoperative Schmerzstärke nach einem Jahr 12,6 $\pm 2,9$ Punkte. Im Vergleich mit anderen Durom-Cup-Studien, die an Patienten mit einer rheumatoiden Arthritis durchgeführt wurden, zeigte sich, dass sowohl die präoperativen Schmerzen als auch die durch die Operation erzielte Schmerzreduktion der eigenen Patienten geringer ausfielen: Bei der Nachuntersuchung empfand die Mehrzahl unserer Patienten mit einer Omarthrose noch zumindest leichte Schmerzen. Auffallend ist, dass in der Untersuchung von Fink et al. (2004) alle Patienten bei der Abschlussuntersuchung nach 36 Monaten schmerzfrei waren. Ein Zusammenhang zum Befund an der Rotatorenmanschette erscheint wenig wahrscheinlich, da die Schmerzfreiheit bei den Patienten mit intakter Rotatorenmanschette nach 2 Jahren, bei den Patienten mit partiellem oder repariertem Defekt der Rotatorenmanschette nach 3 Jahren und bei Patienten mit massivem Defekt der Rotatorenmanschette nach einem Jahr eingetreten war. In der Studie von Fürst et al. (2007) mit einer durchschnittlichen Nachbeoachtungsdauer von 6 Jahren war bei den Patienten mit einer rheumatoiden Arthritis ebenfalls kein Zusammenhang zur Schwere der Erkrankung erkennbar, und bei einer Reihe von Patienten blieben leichte Schmerzen im postoperativen Verlauf über Jahre erhalten. Dagegen sahen Mullett et al. (2007) einen Zusammenhang zum präoperativen Befund: Postoperativ betrug die Schmerzstärke bei Patienten mit einer primären Omarthritis unterschiedlicher Genese durchschnittlich 13,8 Punkte, bei Patienten mit einem Defekt der Rotatorenmanschette dagegen 9,4 Punkte.

Die Arbeitsgruppe Levy beobachtete bei Patienten mit einer Omarthrits eine Besserung der Schmerzen von 1,4 auf 10,6 Punkte (Levy und Copeland, 2001), 3,9 auf 12 Punkte (Levy und Copeland, 2004) bzw. 3,9 \pm 13,7 Punkte (Thomas et al., 2005) und bei Patienten mit einer rheumatoiden Arthritis von 1,6 auf 13,3 Punkte (Levy und Copeland, 
2001), 1,6 auf 11,0 Punkte (Levy et al., 2004) bzw. 1,6 auf 11,2 Punkte (Thomas et al., 2005). Es ist letztlich keine Aussage darüber möglich, ob das Schmerzempfinden bei Patienten mit einer rheumatoiden Arthritis oder einer Omarthrose grundsätzlich durch das Einsetzen eines Oberflächenersatzes differierend beeinflusst wird. Es erscheint interessant, diesem Gesichtspunkt im Rahmen weiterer Untersuchungen nachzugehen.

Als mögliche Einflussgrößen auf die Schmerzentwicklung wurden in der vorliegenden Untersuchung Alter und Geschlecht der Patienten herangezogen. Hierbei beeinflusste das Geschlecht die Schmerzstärke nicht. Allerdings erfuhren Patienten im Alter über 68 Jahren eine signifikant größere Schmerzlinderung als jüngere Patienten. Auch Fakler et al. (2010) beobachteten eine signifikant deutlichere Schmerzreduzierung bei ihren Patienten über 68 Jahren. Als Begründung hierfür nannten sie, dass bei den jüngeren Patienten eine wesentlich größere Rate an Voroperationen bestanden hatte. Diese Begründung kann für unsere Patienten allerdings nicht herangezogen werden. Hier waren in der Anamnese lediglich bei 5 Patienten als Voroperation subakromiale Dekompressionen genannt worden, die sich gleichmäßig auf die Altersgruppen unter $(\mathrm{n}=$ 3) und über $(n=2) 68$ Jahren verteilten.

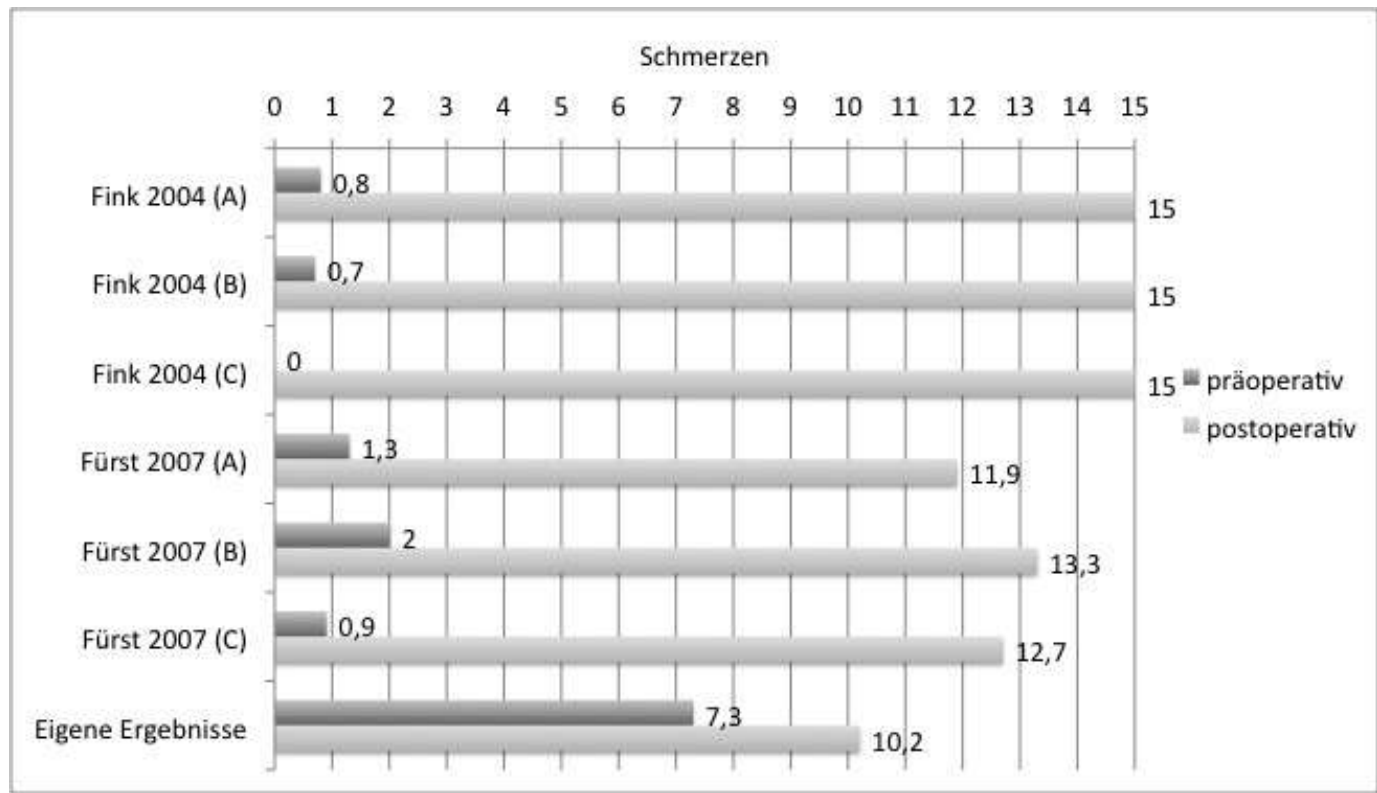

Abbildung 22: Entwicklung der Schmerzstärke der operierten Schultergelenke mit einem Durom-Cup-Oberflächenersatz - Vergleich der eigenen Ergebnisse mit Litera- 
turangaben (Angabe in Punkten: $0=$ schwere Schmerzen, $15=$ keine Schmerzen)

Zur Schulterfunktion existiert eine Reihe von Beurteilungsschlüsseln, von denen der Constant-Score nach Constant und Murley (1987) am gebräuchlichsten ist. Er erlaubt eine umfassende Beurteilung der Schulterfunktion anhand objektiver (Kraft, Beweglichkeit) und subjektiver (Schmerz, Alltagsaktivitäten) Befunde. Im Rahmen der eigenen Untersuchung besserte sich der Constant-Score statistisch signifikant von eingangs 44,6 \pm 10,6 Punkten auf postoperativ 61,4 \pm 13,1 Punkte. Der direkte Vergleich mit anderen Studien zum Durom-Cup erbrachte eine sehr gute Übereinstimmung (Abbildung 23). Da in den anderen Studien hautpsächlich Patienten mit rheumatoider Arthritis behandelt wurden, lässt dieses Ergebnis den Schluss zu, dass im Hinblick auf die Funktionalität der Schulter nach Einsetzen des Durom-Cups kein deutlicher Einfluss der Grunderkrankung nachweisbar ist.

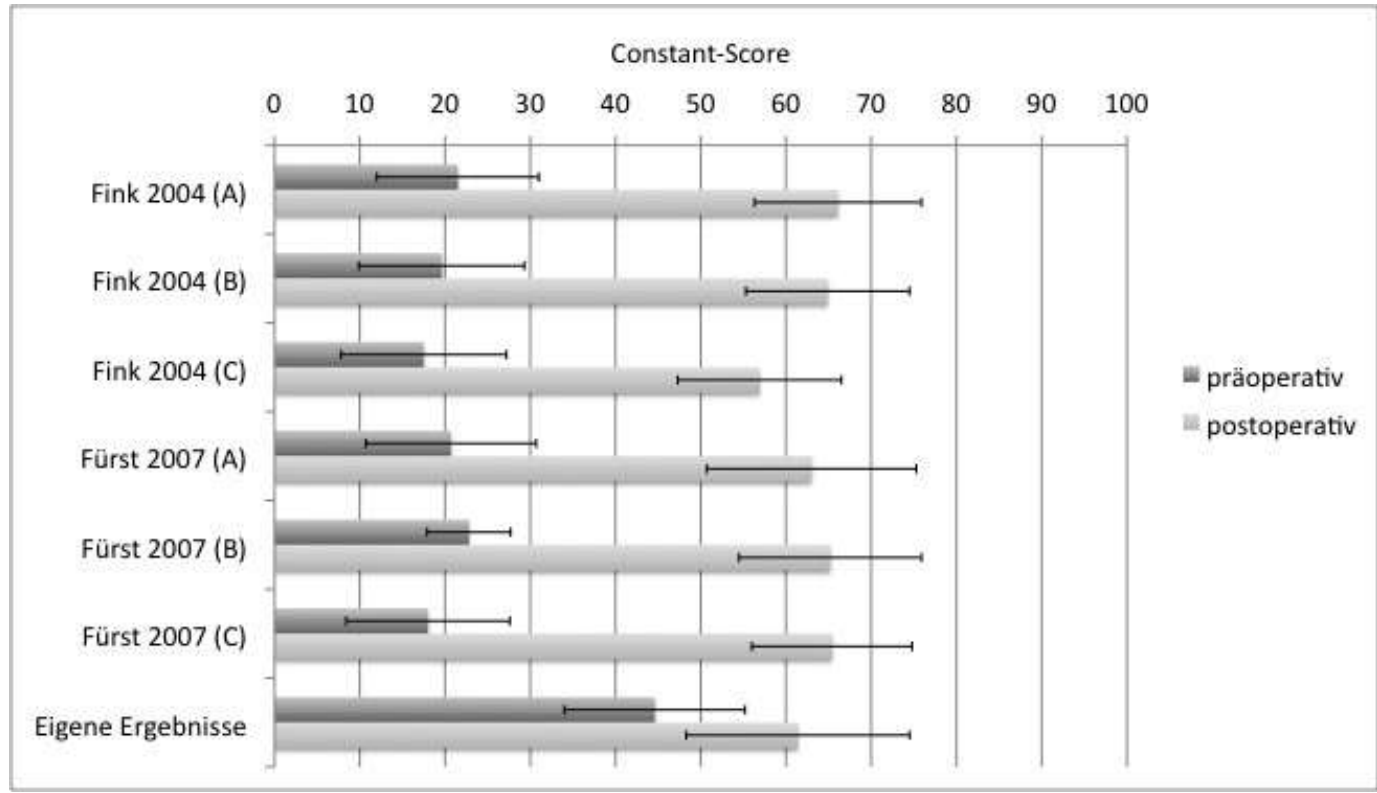

Abbildung 23: Entwicklung des Constant-Scores der operierten Schultergelenke mit einem Durom-Cup-Oberflächenersatz - Vergleich der eigenen Ergebnisse mit Literaturangaben (bestmögliches Ergebnis: 100 Punkte)

Der direkte Vergleich der publizierten Ergebnisse zum Oberflächenersatz bei Patienten mit einer Omarthrose zeigt gleichwertige funktionellen Ergebnisse bei der Verwendung 
eines Copeland- oder DUROM-Cups (Abbildung 24). Auch für andere Indikationen als der Omarthrose oder der rheumatoiden Arthritis lagen die funktionellen Ergebnisse in einem ähnlichen Bereich (Alund et al., 2000; Levy et al., 2004; Raiss et al., 2009; Fakler et al., 2010; Raiss et al., 2010). Eine Ausnahme bildet auch hier die Studie von Mullett et al. (2007): Bei Patienten mit einer primären Osteoarthritis erzielten sie durch Einsetzen eines Copeland-Cups eine Besserung des Constant-Scores von 11,5 auf 62,1 Punkte, bei Patienten mit Defekten der Rotatorenmanschette dagegen nur von 6,6 auf 29,4 Punkte. Den Einfluss des präoperativen Zustandes der Rotatorenmanschette auf das funktionelle Endergebnis konnten Fink et. (2004) sowie Fürst et al. $(2007,2008)$ nicht bestätigen.

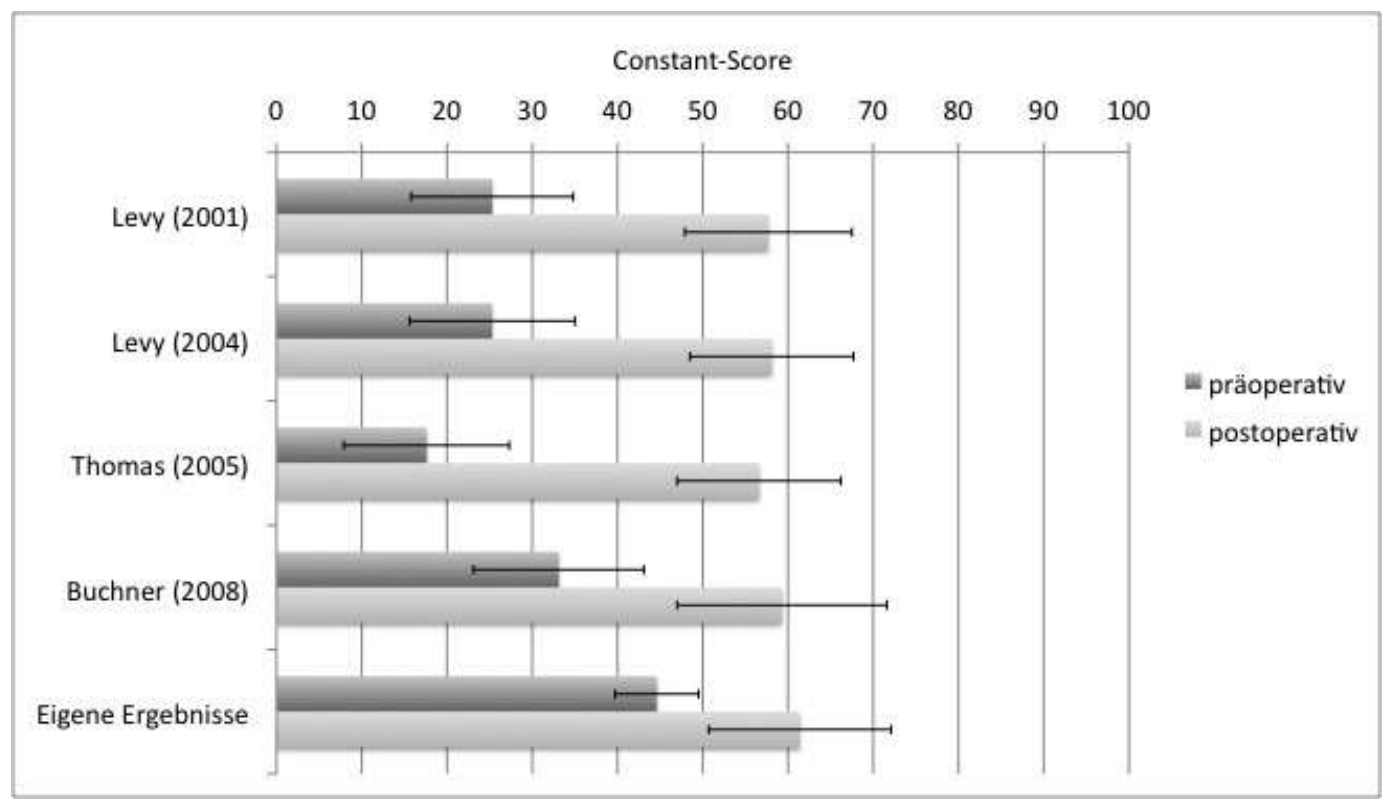

Abbildung 24: Entwicklung des Constant-Scores bei Patienten mit einer Omarthrose - Vergleich der eigenen Ergebnisse mit Literaturangaben (bestmögliches Ergebnis: 100 Punkte)

Gerade bei massiven Schäden der Rotatorenmanschette kann der Oberflächenersatz gegenüber einer Totalendoprothese gewisse Vorteile bieten: Beispielsweise kann durch eine geringe Modifikation bei der Implantation des Cups in Valgusstellung eine Artikulation mit dem Akromion erzielt werden, die mit einer Schaftprothese nicht not- 
wendigerweise erreicht wird. Auf diese Weise können die Zielkriterien Schmerzfreiheit, $90^{\circ}$ Abduktion und $20^{\circ}$ Außenrotation (Neer et al., 1982) auch bei Schultergelenksarthrosen mit begleitenden Defekten der Rotatorenmanschette erfüllt werden (Jerosch et al., 2008).

Unabhängig von der Operationsindikation sowie dem Zustand der Rotatorenmanschette fielen in allen vergleichenden Studien die funktionellen Ergebnisse des Oberflächenersatzes und der Totalendoprothesen gleichwertig aus (Levy und Copeland, 2004; Levy et al., 2004; Mullett et al., 2007; Buchner et al., 2008).

Bei der Untersuchung des Behandlungserfolges kommt der subjektiven Einschätzung des Patienten beziehungsweise seiner Zufriedenheit mit dem Ergebnis besondere Bedeutung zu. Gegenüber einfachen Beurteilungsinstrumenten wie beispielsweise visuellen Analogskalen bietet der komplexe DASH-Score (Disabilities of the Arm, Shoulder and Hand, Hudak et al., 1996) dem Patienten die Möglichkeit, das Operationsergebnis sowohl global als auch hinsichtlich seiner Erwartungshaltungen zu Schmerzen und Funktion der operierten Schulter subjektiv zu bewerten.

In der vorliegenden Untersuchung beurteilten 21 Patienten (65,7\%) ihr Operationsergebnis mit Hilfe des DASH-Scores subjektiv als sehr gut oder gut und die restlichen 11 Patienten (34,4\%) als befriedigend oder ausreichend. Im Einzelnen wurden die Erwartungen der Patienten hinsichtlich der Schmerzreduzierung bei 90,6\% erfüllt bzw. bei $62,5 \%$ sogar übertroffen. Hinsichtlich der Funktion wurden die Erwartungen bei 93,7\% erfüllt bzw. ebenfalls bei $62,5 \%$ übertroffen. Zusammenfassend lässt sich auf der Basis dieser Resultate feststellen, dass die weit überwiegende Mehrheit der Patienten mit dem Operationsergebnis zufrieden waren. Leider existieren nur wenige Vergleichsangaben in der Literatur. Den DASH-Score setzten nur Fakler et al. (2010) ein: Die subjektive Beurteilung des Behandlungsergebnisses fiel bei ihren Patienten mit einer rheumatoiden Arthritis geringfügig besser aus als in der vorliegenden Untersuchung (1,9 \pm 1,4 Punkte vs. 2,3 $\pm 1,0$ Punkte). Dafür waren die Ergebnisse hinsichtlich der Erwartungshaltung Schmerzen etwas schlechter (2,6 \pm 1,3 Punkte vs. 2,2 \pm 0,9 Punkte) und hinsichtlich der Erwartungshaltung Funktion gleich $(2,4 \pm 1,3$ vs. $2,3 \pm 0,8)$. Mullett et al. (2007) setzten zur Beurteilung der Patientenzufriedenheit eine visuelle Analogskala ein. Danach vergaben ihre Patienten mit einer primären Omarthritis nach Implantation eines Copeland-Cups im Mittel 8,5 von 10 Punkten und Patienten mit einem Defekt der Rotatorenmanschette 7,3 von 10 Punkten. In der Studie von Raiss et 
al. 2009/2010 waren nach Implantation eines Copeland-Cups $81 \%$ der Patienten zufrieden oder sehr zufrieden, 15\% unentschlossen und 4\% enttäuscht. Mit dem Ergebnis nach Einsetzen eines Scan-Cups waren in der Studie von Alund et al. (2000) nur $79 \%$ der Patienten zufrieden. In der Studie von Rydholm und Sjögren (1993) zeigten sich $94 \%$ zufrieden mit dem Ergebnis und $82 \%$ der Patienten gaben an, die Implantation der Scan-Shoulder habe zu einer Besserung ihrer Symptomatik geführt.

Auffallend ist, dass in der vorliegenden Untersuchung bei 84,6\% der Männer, aber nur bei 47,4\% der Frauen die Funktion der operierten Schulter besser als erwartet ausfiel, während das Alter im Gegensatz zur Untersuchung von Fakler et al. (2010) hier keinen Einfluss ausübte. Ob allgemein bei Frauen eine größere Anspruchshaltung gegenüber dem Operationsergebnis besteht, lässt sich anhand der eigenen Untersuchung nicht beantworten. Dass dieser geschlechtsspezifische Unterschied isoliert für die Erwartungshaltung Funktion und für keinen anderen der hier untersuchten Parameter auftrat, lässt eher einen Zufallsbefund vermuten. Eine Klärung dieser Frage könnten nur weitere Studien an größeren Fallzahlen herbeiführen.

Zum Überleben des Oberflächenersatzes liegen im Rahmen der publizierten Fallserien zahlreiche Einzelbefunde vor, die auf geringe Komplikationsraten und ein langfristiges Überleben der Prothesen hinweisen. Eine Ausnahme stellen die Erfahrungen mit der Scan-Shoulder dar. Sowohl in der Studie von Rydholm und Sjögren (1993) als auch in der Studie von Alund et al. (2000) waren bei der radiologischen Nachuntersuchung bei jeweils $25 \%$ der Prothesen Lockerungszeichen in Form von Veränderungen des Inklinationswinkels oder Positionsänderungen des Scan-Cups erkennbar. Diese hohe Rate an Lockerungen wurde in der Untersuchung von Rydholm und Sjögren auf einen hohen Anteil an Patienten mit massiven Strukturverlusten am Humeruskopf zurückgeführt. Jedoch berichtete Alund nicht von vergleichbaren Oberflächendefekten. Am wahrscheinlichsten ist die hohe Lockerungsrate der schlechten Verankerung der ScanCups geschuldet, die außer ihrem Innenrelief keine weiteren Befestigungselemente aufweisen.

Dagegen können die Lockerungsraten sowohl bei Copeland- als auch bei DUROMCups als gering bezeichnet werden. Im eigenen Patientengut zeigten sich nur bei einem Patienten Lockerungszeichen in Form eines Lysesaums bei einem Humeruskopf- 
Hochstand und unauffälligem klinischem Befund. Bei zwei Patientinnen wurde ein isolierter Humeruskopf-Hochstand ohne weitere radiologische oder klinische Befunde gesehen, so dass eine drohende Lockerung fraglich erscheint. Ein Fortschreiten der Omarthrose deutete sich bei drei weiteren Patienten durch glenoidale Sklerosierungen bzw. eine periartikuläre Ossifikation an. Eine Revision war bei keinem unserer Patienten erforderlich. Im Rahmen der Patientenauswahl für die vorliegende Untersuchung war allerdings eine Patientin mit Omarthrose und Ruptur im Bereich der Rotatorenmanschette aufgefallen, bei der im Jahr 2006 die Implantation eines DUROM-Cups erfolgt und im Jahr 2008 wegen persistierender Schmerzen eine Delta-InverseProthese eingesetzt worden war. Da diese Patientin zum Zeitpunkt der Nachuntersuchung keinen DUROM-Cup-trug, war sie aus der vorliegenden Untersuchung ausgeschlossen worden.

Im Patientengut von Fakler et al. (2010) fand sich in keinem Fall ein radiologischer Hinweis auf eine Migration des Oberflächenersatzes. Bei einem Patienten mit einem röntgenologisch erkennbaren Lysesaum bei deutlich eingeschränkter Funktion war die Indikation zur Revision mit Prothesenwechsel gegeben. Fürst et al. (2009) berichteten von einer Patientin, bei der der Durchmesser des DUROM-Cups zunächst zu groß gewählt worden war. Durch eine Revision mit Wechsel auf einen kleineren Prothesendurchmesser konnte eine völlige Beschwerdefreiheit erzielt werden. Bei zwei weiteren Patienten war die zusätzliche Implantation einer glenoidalen Komponente wegen des Auftretens von Erosionen erforderlich. Bei einem Patienten, der neben Erosionen einen Humeruskopfhochstand aufwies, wurde auf Grund der Beschwerdefreiheit keine Revison durchgeführt. Als Sonderfall fiel bei der Nachuntersuchung eine Patientin mit einer ausgeheilten subcapitalen Humerusfraktur der operierten Schulter auf. Die Frau hatte sich nach einem schweren Sturz auf den operierten Arm nicht in ärztliche Behandlung begeben und war bei der Nachuntersuchung beschwerdefrei. In der Untersuchung von Fink et al. (2004) wies bei zwei Röntgenkontrollen drei und 36 Monate post operationem kein Patient Abweichungen vom radiologischen Normalbefund auf.

Die Erfahrungen mit dem Copeland sind vergleichbar gut. In allen ausgewerteten Studien wurde von jeweils ein bis zwei Revisionen beziehungsweise dem Wechsel auf eine Totalprothese berichtet. Als Indikationen für die Reoperation wurden übereinstimmen schmerzhafte Funktionseinschränkungen, radiologisch erkennbare Locke- 
rungszeichen oder ein Voranschreiten von Arthrosen beziehungsweise Übergreifen auf den glenoidalen Bereich genannt.

Aus den in der Literatur beschriebenen sowie den eigenen Erfahrungen mit dem Oberflächenersatz des Schultergelenks kann der Schluss gezogen werden, dass der hier verwendete DUROM-Cup besonders bei Patienten mit einer Omarthrose oder rheumatoiden Arthritis eine gute Verankerung gewährleistet und eine funktionelle Wiederherstellung der geschädigten Humerusoberfläche ermöglicht. Voraussetzung dafür ist eine erhaltene Rotatorenmanschette, obwohl in der Literatur beschrieben wurde, dass der Oberflächenersatz auch bei geschädigter Rotatorenmanschette bei leicht valgischem Einsetzen möglich ist (Jerosch et al., 2008).

Das Risiko von Komplikationen beziehungsweise Prothesenlockerungen ist beim Oberflächenersatz des Humeruskopfes gering. Die funktionellen Ergebnisse und die erzielte Schmerzfreiheit sind bei beiden Prothesentypen gleich gut und den Resultaten nach Implantation einer Totalendoprothese nicht unterlegen. Daher lassen sich mit Hilfe des Oberflächenersatzes auch die Erwartungen der Patienten zu deren Zufriedenheit erfüllen. 


\section{$6 \quad$ Zusammenfassung}

Bei 29 Patienten (16 Frauen, 13 Männer) im Alter von 70,2 $\pm 7,1$ Jahren (55 bis 83 Jahre) wurde an insgesamt 32 Schultergelenken ein humeraler Oberflächenersatz mittels Durom-Cup-Prothese vorgenommen. Bei 93,8\% der Schultergelenke lag eine primäre Omarthrose und bei jeweils $3,1 \%$ eine posttraumatische Omarthrose oder eine Humeruskopfnekrose vor. Nach durchschnittlich 2,7 $\pm 2,1$ Jahren $(0,5$ bis 9 Jahre) wurde eine klinische und radiologische Nachuntersuchung vorgenommen.

Der mittlere Constant-Score betrug präoperativ 44,6 $\pm 10,6$ und postoperativ $61,4 \pm 13,1$ Punkte, d.h. es hatte eine hoch signifikante Verbesserung um $16,8 \pm 10,5$ Punkte stattgefunden. Die Besserungen betrafen alle Unterkategorien des Constant-Scores (Schmerzen, Aktivität, Beweglichkeit und Kraft).

Nach dem modifzierten DASH-Score beurteilten 21 Patienten (65,7\%) ihr Operationsergebnis subjektiv als sehr gut oder gut und 11 Patienten (34,4\%) als befriedigend oder ausreichend. Die Erwartungen der Patienten hinsichtlich der Schmerzreduzierung wurden bei $90,6 \%$ erfüllt bzw. bei $62,5 \%$ übertroffen. Hinsichtlich der Funktion wurden die Erwartungen bei 93,7\% erfüllt bzw. ebenfalls bei 62,5\% übertroffen.

Geschlecht und Alter waren nicht mit den funktionellen Ergebnissen korreliert. Als Ausnahme erfuhren Patienten im Alter über 68 Jahren eine größere Schmerzlinderung (3,6 \pm 1,8 Punkte) als jüngere Patienten (1,8 $\pm 2,2$ Punkte, $p<0,05)$.

Radiologisch zeigten sich bei jeweils zwei Patienten/Schultergelenken eine ausgeprägte Sklerosierung im Glenoid-Bereich oder ein Humeruskopf-Hochstand und bei jeweils einem Patienten eine periartikuläre Ossifikation oder Lockerungszeichen mit Lysesaum-Bildung und Humeruskopf-Hochstand. Eine Revision bzw. ein Prothesenwechsel war bei keinem Patienten erforderlich.

Bei einem Literaturvergleich waren die eigenen Ergebnisse vergleichbar gut wie die Ergebnisse anderer Autoren mit dem DUROM- oder dem Copeland-Cup. Der Behandlungserfolg des Oberflächenersatzes war von einer Omarthrose oder rheumatoiden Arthritis als Grunderkrankung unabhängig. Die Resultate des vergleichsweise komplikationslosen Oberflächenersatzes waren denjenigen nach Implantation einer Totalendoprothese nicht unterlegen. 


\section{$7 \quad$ Anhang}

\subsection{Abkürzungen}

$\begin{array}{ll}\text { DASH } & \text { Disabilities of the Arm, Shoulder and Hand } \\ \text { HKN } & \text { Humeruskopfnekrose } \\ \text { k.A. } & \text { Instabilität } \\ \text { Mon. } & \text { keine Angabe } \\ \text { MW } & \text { Monat(e) } \\ \text { n } & \text { Mittelwert } \\ \text { NU } & \text { Anzahl der Werte } \\ \text { OA } & \text { Nachuntersuchung } \\ \text { OP } & \text { Omarthrose } \\ \text { p } & \text { Operation(en) } \\ \text { PA } & \text { Signifikanzniveau } \\ \text { Pat. } & \text { Posttraumatische Arthrose } \\ \text { RA } & \text { Patienten } \\ \text { RM } & \text { Rheumatoide Arthritis } \\ \text { SAD } & \text { Rotatorenmanschette } \\ \text { SD } & \text { Arthroskopische subacromiale Dekompression } \\ \text { SG } & \text { Standardabweichung } \\ \text { TEP } & \text { Schultergelenk(e) } \\ & \end{array}$




\section{$8 \quad$ Literaturverzeichnis}

Alund M, Hoe-Hansen C, Tillander B, Heden BA, Norlin R (2000): Outcome after cup hemiarthroplasty in the rheumatoid shoulder: a retrospective evaluation of 39 patients followed for 2-6 years. Acta Orthop Scand 71, 180-184

Baer WS (1918): Arthroplasty with the aid of animal membrane. Amer J Orthop Surg $16,1-8$

Barrett WP, Franklin JL, Jackins SE, Wyss CR, Matsen FA, 3rd (1987): Total shoulder arthroplasty. J Bone Joint Surg Am 69, 865-872

Blauth W, Donner K (1979): Zur Geschichte der Arthroplastik. Z Orthop Ihre Grenzgeb $117,997-1006$

Boileau P, Sinnerton RJ, Chuinard C, Walch G (2006): Arthroplasty of the shoulder. J Bone Joint Surg $\mathrm{Br} 88,562-575$

Boileau P, Walch G (1997): The three-dimensional geometry of the proximal humerus. Implications for surgical technique and prosthetic design. J Bone Joint Surg Br $79,857-865$

Brown JE, Mc GW, Shaw DT (1958): Use of cutis as an interposing membrane in arthroplasty of the knee. J Bone Joint Surg Am 40-A, 1003-1018

Buchner M, Eschbach N, Loew M (2008): Comparison of the short-term functional results after surface replacement and total shoulder arthroplasty for osteoarthritis of the shoulder: a matched-pair analysis. Arch Orthop Trauma Surg 128, 347-354

Buck FM, Jost B, Hodler J (2008): Shoulder arthroplasty. Eur Radiol 18, 2937-2948

Burgess DL, McGrath MS, Bonutti PM, Marker DR, Delanois RE, Mont MA (2009): Shoulder resurfacing. J Bone Joint Surg Am 91, 1228-1238

Cofield RH (1979): Total joint arthroplasty. The shoulder. Mayo Clin Proc 54, 500-506 
Cofield RH (1984): Total shoulder arthroplasty with the Neer prosthesis. J Bone Joint Surg Am 66, 899-906

Constant CR, Murley AH (1987): A clinical method of functional assessment of the shoulder. Clin Orthop Relat Res 160-164

Fakler JK, Gresens M, Müller C, Melzer C (2010): 2- bis 6-Jahresergebnisse eines zementierten humeralen Oberflächenersatzes (DUROM Cup) an der Schulter. Orthopädische Praxis 46, 385-391

Fenlin JM, Jr., Ramsey ML, Allardyce TJ, Frieman BG (1994): Modular total shoulder replacement. Design rationale, indications, and results. Clin Orthop Relat Res $37-46$

Fenlin JM, Jr., Vaccaro A, Andreychik D, Lin S (1990): Modular total shoulder: early experience and impressions. Semin Arthroplasty 1, 102-111

Fink B, Singer J, Lamla U, Ruther W (2004): Surface replacement of the humeral head in rheumatoid arthritis. Arch Orthop Trauma Surg 124, 366-373

Fürst M, Fink B, Rüther W (2007): The DUROM cup humeral surface replacement in patients with rheumatoid arthritis. J Bone Joint Surg Am 89, 1756-1762

Fürst M, Fink B, Rüther W (2008): The DUROM cup humeral surface replacement in patients with rheumatoid arthritis. Surgical technique. J Bone Joint Surg Am 90 Suppl 2 Pt 2, 287-298

Gartsman GM, Russell JA, Gaenslen E (1997): Modular shoulder arthroplasty. J Shoulder Elbow Surg 6, 333-339

Godeneche A, Boileau P, Favard L, Le Huec JC, Levigne C, Nove-Josserand L et al. (2002): Prosthetic replacement in the treatment of osteoarthritis of the shoulder: early results of 268 cases. J Shoulder Elbow Surg 11, 11-18

Habermeyer P, Ebert T (1999): Aktueller Entwicklungsstand und Perspektiven der Schulterendoprothetik. Unfallchirurg 102, 668-683 
Hawkins RJ, Bell RH, Jallay B (1989): Total shoulder arthroplasty. Clin Orthop Relat Res 188-194

Hudak PL, Amadio PC, Bombardier C (1996): Development of an upper extremity outcome measure: the DASH (disabilities of the arm, shoulder and hand) [corrected]. The Upper Extremity Collaborative Group (UECG). Am J Ind Med $29,602-608$

Irlenbusch U, Berth A, Blatter G, Zenz P (2011): Variability of medial and posterior offset in patients with fourth-generation stemmed shoulder arthroplasty. Int Orthop

Jerosch J, Heisel J (2003): Schulterendoprothetik: eine Standortbestimmung. Dt Ärztebl 100, A2366-2377

Jerosch J, Schunck J, Morsy MG (2008): Oberflächenersatz bei Rotatorenmanschettendefektarthropathie mit erhaltener Subscapularisfunktion. Z Orthop Unfall 146, 206-210

Jonsson E, Brattstrom M, Lidgren L (1988): Evaluation of the rheumatoid shoulder function after hemiarthroplasty and arthrodesis. Scand J Rheumatol 17, 17-26

Jonsson E, Egund N, Kelly I, Rydholm U, Lidgren L (1986): Cup arthroplasty of the rheumatoid shoulder. Acta Orthop Scand 57, 542-546

Kolbel R, Friedebold G (1973): Möglichkeiten der Alloarthroplastik an der Schulter. Arch Orthop Unfallchir 76, 31-39

Kolbel R, Friedebold G (1975): Schultergelenkersatz. Z Orthop Ihre Grenzgeb 113, $452-454$

König F (1912): Über Implantation von Elfenbein zum Ersatz von Knochen- und Gelenkenden. Verh dtsch Ges Chir 41, 41-43

König F (1913): Über die Implantation von Elfenbein zum Ersatz von Knochen- und Gelenkenden. Beitr klin Chir 85, 91-114 
Langenbeck von B (1974): Über die Endresultate der Gelenkresektionen im Kriege. Langenbecks Arch klin Chir 16, 340-356

Lettin AW (1980): Total joint replacement. Br J Hosp Med 24, 328, 330, 333-324 passim

Lettin AW, Copeland SA, Scales JT (1982): The Stanmore total shoulder replacement. J Bone Joint Surg Br 64, 47-51

Levy O, Copeland SA (2001): Cementless surface replacement arthroplasty of the shoulder. 5- to 10-year results with the Copeland mark-2 prosthesis. J Bone Joint Surg Br 83, 213-221

Levy O, Copeland SA (2004): Cementless surface replacement arthroplasty (Copeland CSRA) for osteoarthritis of the shoulder. J Shoulder Elbow Surg 13, 266-271

Levy O, Funk L, Sforza G, Copeland SA (2004): Copeland surface replacement arthroplasty of the shoulder in rheumatoid arthritis. J Bone Joint Surg Am 86-A, $512-518$

Lexer E (1908a): Freie Knochenplastik, Gelenksteifigkeit, Gelenktransplantation. Arch klin Chir 86, 939-947

Lexer E (1908b): Über Gelenktransplantationen. Langenbecks Arch klin Chir 90, 263268

Lexer E (1917): Die Beweglichmachung versteifter Gelenke mit und ohne Gewebszwischenlagerung. Zbl Chir 44, 2-7

Marquardt W, Steinbeck J: Oberflächenersatz. In: AE-Manual der Endoprothetik. Schulter.; hrsg. v. Loew M; Springer, Heidelberg 2010, S. 40-45

Marquardt W, Witt KA, Steinbeck J: Schulterendoprothetik. In: Endoprothetik; hrsg. v. Krukemeyer MG, Möllenhoff G; de Gruyter, Berlin 2009, S. 30-62

McElwain JP, English E (1987): The early results of porous-coated total shoulder arthroplasty. Clin Orthop Relat Res 217-224 
Mullett H, Levy O, Raj D, Even T, Abraham R, Copeland SA (2007): Copeland surface replacement of the shoulder. Results of an hydroxyapatite-coated cementless implant in patients over 80 years of age. J Bone Joint Surg Br 89, 1466-1469

Murphy JB (1913): Arthroplasty. Ann Surg 57, 593-600

Neer CS, 2nd (1974): Replacement arthroplasty for glenohumeral osteoarthritis. J Bone Joint Surg Am 56, 1-13

Neer CS, 2nd (2011): The classic: Articular replacement for the humeral head. 1955. Clin Orthop Relat Res 469, 2409-2421

Neer CS, 2nd, Watson KC, Stanton FJ (1982): Recent experience in total shoulder replacement. J Bone Joint Surg Am 64, 319-337

Nwakama AC, Cofield RH, Kavanagh BF, Loehr JF (2000): Semiconstrained total shoulder arthroplasty for glenohumeral arthritis and massive rotator cuff tearing. J Shoulder Elbow Surg 9, 302-307

Payr E (1910): Gelenksteifen und Gelenkplastik. Verh dtsch Ges Chir 41, 516-523

Pean JE (1894): Des moyens prosthetique destines a obtenir la reparation de parties osseuses. (Nachdruck: Clin Orthop Relat Res [1973], 94: 4-7). Gaz de Hop Paris 67, 291-302

Pospeschill M: Statistische Methoden; Elsevier, Heidelberg 2006

Post M, Haskell SS, Jablon M (1980): Total shoulder replacement with a constrained prosthesis. J Bone Joint Surg Am 62, 327-335

Raiss P, Kasten P, Baumann F, Moser M, Rickert M, Loew M (2009): Treatment of osteonecrosis of the humeral head with cementless surface replacement arthroplasty. J Bone Joint Surg Am 91, 340-349

Raiss P, Pape G, Becker S, Rickert M, Loew M (2010): Der zementfreie Oberflächenersatz bes Humeruskopfes bei Patienten unter 55 Jahren. Orthopäde 39, 201-208 
Reeves B, Jobbins B, Flowers F, Dowson D, Wright V (1972): Some problems in the development of a total shoulder endo-prosthesis. Ann Rheum Dis 31, 425-426

Reimers TC (1970): Zur geschichtlichen Entwicklung gelenkplastischer Eingriffe. Chir plast reconstr $7,2-9$

Robertson DD, Yuan J, Bigliani LU, Flatow EL, Yamaguchi K (2000): Threedimensional analysis of the proximal part of the humerus: relevance to arthroplasty. J Bone Joint Surg Am 82-A, 1594-1602

Rüther W (2002): Durom® Shoulder Cup - Operationstechnik. Fa. Zimmer Germany, Freiburg.

Rydholm U, Sjögren J (1993): Surface replacement of the humeral head in the rheumatoid shoulder. J Shoulder Elbow Surg 2, 286-295

Sachs L: Angewandte Statistik. 11. Aufl.; Springer, Berlin 2004

Scalise JJ, Miniaci A, lanotti J (2008): Resurfacing arthroplasty of the humerus: Indications, surgical technique and clinical results. Curr Orthop Pract 19, 443450

Steffee AD, Moore RW (1984): Hemi-resurfacing arthroplasty of the shoulder. Contemp Orthop 9, 51-59

Thomas SR, Wilson AJ, Chambler A, Harding I, Thomas M (2005): Outcome of Copeland surface replacement shoulder arthroplasty. J Shoulder Elbow Surg $14,485-491$

Torchia ME, Cofield RH, Settergren CR (1997): Total shoulder arthroplasty with the Neer prosthesis: long-term results. J Shoulder Elbow Surg 6, 495-505

Walch G, Boileau P (1999): Prosthetic adaptability: a new concept for shoulder arthroplasty. J Shoulder Elbow Surg 8, 443-451

Wretenberg PF, Wallensten R (1999): The Kessel total shoulder arthroplasty. A 13- to 16-year retrospective followup. Clin Orthop Relat Res 100-103 
Zhang W, Mow CS, Wiedel JD (2000): Unconstrained shoulder arthroplasty. Chin J Traumatol 3, 72-75

Zippel J (1972): [Complete shoulder joint prosthesis of plastic and metal]. Biomed Tech (Berl) 17, 87-91

Zippel J (1973): Arthroplastik des Schultergelenkes. Orthopäde 2, 107-109 


\section{$9 \quad$ Danksagung}

Ich möchte mich hiermit bei Herrn Prof. Dr. med. Rolf Haaker, Leiter der Abteilung für Orthopädie und Traumatologie des St. Vincenz Hospitals Brakel, bedanken, der mir die Möglichkeit gegeben hat, diese Arbeit unter seiner Leitung durchzuführen.

Besonderen Dank auch an die gesamte Arbeitsgruppe für die freundschaftliche Arbeitsatmosphäre, viele wertvolle Anregungen und stete Hilfsbereitschaft, die wesentlich zum Gelingen dieser Arbeit beigetragen haben.

Bei meinen Eltern, meiner Frau und meinen Kindern möchte ich mich ganz besonders herzlich bedanken für die uneingeschränkte, liebevolle und vielseitige Unterstützung während meines Studiums, ohne die diese Arbeit nicht möglich gewesen wäre. 


\section{Lebenslauf}

Name:

Vorname:

Geburtsort:

Geburtsdatum:

Familienstand:

1980-1987

1987-1990

1990-1993

1993-1999

1997

01.02.2000-01.10.2003

2001-05.05.2003

01.12.2003-14.03.2004

15.03.2004-31.03.2008

01.04 .2008

25.09.2010
Saad

Fouzi

Damaskus

05.05.1976

verheiratet, 2 Söhne

Besuch der Grundschule in Damaskus

Besuch der Mittelschule in Damaskus

Besuchs des Gymnasiums wissenschaftlicher Fachrichtung in Damaskus

Studium der Medizin an der Universität Damaskus, Abschluss als Dr. med. mit dem Prädikat "gut“

6 Monate AiP im französischen Hospital in Damaskus

Tätigkeit in der Notfallabteilung des italienischen Krankenhauses in Damaskus

Assistenzarzt der Abteilung für allgemeine Chrirugie am Militärkrankenhaus in Damaskus

Hospitation in der orthopädischen Abteilung des St. Elisabeth-Hospitals in Gütersloh

Assistenzarzt in der orthopädischen Abteilung des St. Elisabeth-Hospitals in Gütersloh unter Leitung von Prof. Dr. med. Küster und ab dem 01.10.2007 unter der Leitung von PD Dr. med. Boudriot

Assistenzarzt in der orthopädischen und unfallchirurgischen Abteilung des St. Vincenz-Hospitals in Brakel unter Leitung von Prof. Dr. med. Haaker

Anerkennung als Facharzt für Orthopädie 